\title{
Symmetry and Equilibrium States
}

\author{
Huzihiro Araki \\ Research Institute for Mathematical Sciences, Kyoto University, Kyoto 606, Japan \\ Akitaka Kishimoto \\ Department of Physics, Kyoto University, Kyoto 606, Japan
}

\begin{abstract}
Within the general framework of $C^{*}$-algebra approach to mathematical foundation of statistical mechanics, we prove a theorem which gives a natural explanation for the appearance of the chemical potential (as a thermodynamical parameter labelling equilibrium states) in the presence of a symmetry (under gauge transformations of the first kind). As a symmetry, we consider a compact abelian group $G$ acting as *automorphisms of a $C^{*}$-algebra $\mathfrak{A}$ (quasi-local field algebra) and commuting (elementwise) with the time translation automorphisms $\varrho_{t}$ of $\mathfrak{A}$. Under a technical assumption which is satisfied by examples of physical interest, we prove that the set of all extremal $\varrho_{t}$-KMS states $\varphi$ (pure phases) of $G$-fixed-point subalgebra $\mathfrak{A}^{G}$ (quasi-local observable algebra) of $\mathfrak{A}$ satisfying a certain faithfulness condition is in one-to-one correspondence with the set of all extremal $G$-invariant $\varrho_{t} \cdot \alpha_{t}-\mathrm{KMS}$ states $\varphi^{-}$of $\mathfrak{A}$ with $\alpha$ varying over one-parameter subgroups of $G$ (the specification of $\alpha$ being the specification of the chemical potential), where the correspondence is that the restriction of $\varphi^{-}$to $\mathfrak{A l}^{G}$ is $\varphi$.
\end{abstract}

\section{§ 1. Introduction}

In equilibrium statistical mechanics, one of basic problems is how to understand the number of independent thermodynamic variables for equilibrium states of a given system. In the Heisenberg picture, a mathematical description of a system is given in terms of observables and its time translation. So-called KMS condition picks out states labelled by a real parameter $\beta$; they are interpreted as equilibrium states at inverse temperature $\beta$. The justification for such an interpretation has been given by Haag et al. [2], who characterize KMS states by stability under dynamical perturbation and then show that the stability condition is equivalent to the KMS condition under some assumptions.

For given $\beta$, there may be many KMS states which can be labelled by additional macroscopic variables. It is also useful to consider a family of timetranslations labelled by some external parameters (such as external magnetic 
field), which yields KMS-states parametrized by $\beta$ and external parameters, in general. However, there are examples such as gas in infinite continuum, where text-books teach us that equilibrium states are parametrized not only by $\beta$ but also by other parameters such as the chemical potential $\mu$. Moreover, the usual prescription for equilibrium states with given $\beta$ and $\mu$ does not give states satisfying the KMS condition relative to a given time translation: it rather gives states satisfying the KMS condition relative to a modified time translation, the modification being given by a gauge transformation.

The purpose of this paper is to give a natural explanation for the chemical potential as a label of equilibrium states. We consider a system with a symmetry group $G$, which we assume to be compact and abelian. A typical example is the additive group of real numbers modulo $2 \pi$, which acts as gauge transformations of the first kind, changing a field $\psi(x)$ to $e^{i \theta} \psi(x)$. The system is described by the algebra $\mathfrak{A}$ generated by quasi-local fields; its dynamics is described by oneparameter group $\varrho_{t}$ of time translations, which commute with any symmetry transformation $g \in G$.

Under some circumstances, one can take the view-point that only those elements of $\mathfrak{A}$, which are invariant under the (gauge) symmetry transformations, correspond to physical observables, the rest of $\mathfrak{A}$ being a mathematical device. The set $\mathfrak{I}^{\boldsymbol{G}}$ of such elements is invariant (as a set) under the translations $\varrho_{t}$ and the restriction of $\varrho_{t}$ to $\mathfrak{I}^{G}$ is the physical time translations. In this view-point, equilibrium states should be KMS states of $\mathfrak{Q}^{G}$ relative to the restriction of $\varrho_{t}$ to $\mathfrak{2}^{G}$. (We call them simply $\varrho_{t}-\mathrm{KMS}$ states of $\mathfrak{Q}^{G}$.) In particular, extremal $\varrho_{t}$-KMS states of $\mathfrak{Q}^{G}$ are interpreted as pure phases.

In order to study $\varrho_{t}$-KMS states of $\mathfrak{Q}^{G}$, we are entitled to discuss mathematically their extentions to $\mathfrak{A}$ as states. It is an immediate consequence of the compactness of $G$ that each state $\varphi$ of $\mathfrak{Q}^{G}$ has a unique extention to a $G$-invariant state $\varphi^{-}$of $\mathfrak{A}$. It is also immediate that if a state $\varphi^{-}$of $\mathfrak{A}$ satisfies the KMS condition relative to $\varrho_{t} \cdot \alpha_{t}$ for some one-parameter subgroup $\alpha_{t}$ of $G$, then the restriction $\varphi$ of $\varphi^{-}$to $\mathfrak{I}^{G}$ is a $\varrho_{t}$-KMS state of $\mathfrak{Q}^{G}$.

Our main result is that the extention $\varphi^{-}$of a pure phase $\varphi$ of $\mathfrak{Q}^{G}$ is an extremal $G$-invariant $\varrho_{t} \cdot \alpha_{t}-\mathrm{KMS}$ state for some one-parameter subgroup $\alpha_{t}$ of $G$ and that the restriction $\varphi$ of an extremal $G$-invariant $\varrho_{t} \cdot \alpha_{t}$-KMS state $\varphi^{-}$of $\mathfrak{A}$ to $\mathfrak{A}^{G}$ is a pure phase. As a consequence, pure phases of $\mathfrak{Q}^{G}$ are in one-to-one correspondence with extremal $G$-invariant $\varrho_{t} \cdot \alpha_{t}$ states of $\mathfrak{A}$ with $\alpha_{t}$ varying over one-parameter subgroups of $G$, the correspondence being given by restriction and extension. If $G$ is a one-parameter group, say $\gamma_{t}$ with $\gamma_{2 \pi}=$ identity, then $\alpha_{t}=\gamma_{\mu t}$ where the real parameter $\mu$ labelling different one-parameter subgroups is the chemical potential.

It may happen that the extremal $G$-invariant $\varrho_{t} \cdot \alpha_{t}$-KMS state $\varphi^{-}$is not extremal $\varrho_{t} \cdot \alpha_{t}$-KMS state. In that case, the central decomposition of $\varphi^{-}$yields extremal $\varrho_{t} \cdot \alpha_{t}$-KMS states whose restriction to $\mathfrak{A}^{G}$ are all equal to the extremal $\varrho_{t}$-KMS state $\varphi$. Conversely, if $\varphi^{\wedge}$ is an extremal $\varrho_{t} \cdot \alpha_{t}-\mathrm{KMS}$ state, then its restriction $\varphi$ to $\mathfrak{Q}^{G}$ is an extremal $\varrho_{t}$-KMS state of $\mathfrak{Q}^{G}$ and $\varphi^{\wedge}$ is in the central decomposition of the $G$-invariant extention $\varphi^{-}$of $\varphi$ to $\mathfrak{A}$. This describes the situation of a broken symmetry.

Our results described above depends on an assumption on the algebra $\mathfrak{A}$ and its time translation $\varrho_{t}$. The main assumption which is described in detail in the 
next section will be satisfied if the field asymptotically commutes with observables at large space separation and contains non-trivial elements on which $G$ acts as a multiplication of its character such that characters associated with such elements generates the dual of $G$. In addition to these assumptions on the algebra, we also assume a certain faithfulness condition on the state $\varphi$ of $\mathfrak{A}^{G}$. When this assumption for $\varphi$ is violated, $G$ has one-sided spectrum on the cyclic space associated with $\varphi^{-}$, as is discussed in the last section.

A generalization of the present result to a compact non-abelian symmetry group $G$ will be given in a subsequent paper.

Independently the same problem has been studied in [4]. In [4], main assumption on the algebra is asymptotic abelianness relative to automorphisms $\tau^{n}$ commuting with the time translation and gauge transformations.

Our Assumption A has a somewhat stronger requirement in the sense that it requires the existence of an operator $b^{(p)}$ satisfying $b^{(p) *} b^{(p)}+b^{(p)} b^{(p) *} \geqq 1$ and transforming under gauge group $G$ as an irreducible representation $p$ for a set of $p$ generating the dual of $G$. [ $b_{n}^{(p)}$ in Assumption (A) is then taken to be $\left.\tau^{n}\left(b^{(p)}\right)\right]$ In a given model, however, such assumption is easy to check.

On the other hand our Assumption A has a somewhat weaker requirement in the sense that the asymptotic abelianness needed here is between an observable (elements of $\mathfrak{A}_{G}$ ) and an arbitrary element and not between arbitrary elements. This point is the first advantage of the present approach over that in [4].

Our Assumption B is to exclude the case of one-sided.spectrum [cf. Section 9 (2)]. In [4], the case where Assumption B is not satisfied is fully analyzed.

In [4], the state under consideration (restricted to the observable algebra) is assumed to be invariant under $\tau^{n}$ mentioned above. If $\tau$ is time translation, this is automatic but the asymptotic abelianness relative to time is hard to prove in a given model. If $\tau$ is not time translation, then the invariance of the state under $\tau^{n}$ is an assumption. In the present approach, the case of non-invariant state is included. This is the second advantage of the present approach over that in [4].

In [4], the gauge group $G$ is any compact group, while we assumed $G$ to be an abelian compact group here.

\section{§ 2. Main Theorems}

We consider a $C^{*}$-dynamical system, namely a $C^{*}$-algebra $\mathfrak{A}$ with an identity 1 and a one-parameter group of *-automorphisms $\varrho_{t}(t \in \mathbb{R})$ of $\mathfrak{A}$ such that $t \rightarrow \varrho_{t}(x)$ is continuous for each $x \in \mathfrak{A}$. Let $G$ be a compact abelian group of *automorphisms of $\mathfrak{A}$ commuting with $\varrho_{t}, t \in \mathbb{R}$. The set of all elements $x$ of $\mathfrak{A}$ satisfying $g(x)=x$ for all $g \in G$ is denoted by $\mathfrak{A}^{G}$. It is a $C^{*}$-subalgebra of $\mathfrak{A}$ containing 1 . For each unitary character $p$ of $G$ (i.e. $p \in G^{\wedge}$ ), we define

$$
\varepsilon_{p}(x)=\int_{G} p(g)^{*} g(x) d g
$$

where $d g$ is the normalized Haar measure. For the identity $p=1$, we have $\mathfrak{A}^{G}=$ $\varepsilon_{1}(\mathfrak{l})$.

We make the following assumption. 
Assumption A. There exists a sequence $b_{n}^{(p)} \in \varepsilon_{p}(\mathfrak{H})$ for each $p \in \Delta$ for some generating subset $\Delta$ of $G^{\wedge}$, with the following properties:

(a) $\sup _{n}\left\|b_{n}^{(p)}\right\|<\infty$.

(b) $\left(b_{n}^{(p)}\right)^{*} b_{n}^{(p)}+b_{n}^{(p)}\left(b_{n}^{(p)}\right)^{*} \geqq 1$.

(c) $\lim _{n}\left\|\left[b_{n}^{(p)}, x\right]\right\|=0$ for all $x \in \mathfrak{A}^{G}$.

(d) $\lim _{n}\left\|\left[\left(b_{n}^{(p)}\right)^{*} \varrho_{t}\left(b_{n}^{(p)}\right), x\right]\right\|=\lim _{n}\left\|\left[\varrho_{t}\left(b_{n}^{(p)}\right)\left(b_{n}^{(p)}\right)^{*}, x\right]\right\|=0$, for all $x \in \mathfrak{A}$.

(e) $\left\{\varrho_{t}\left(b_{n}^{(p)}\right) ; n=1,2, \ldots\right\}$ is an equicontinuous family of continuous functions of $t$ for each $p \in \Delta$.

For technical reason we also assume the following:

Assumption B. For each $p \in \Delta$,

$$
\sup _{n} s_{c}\left\{\pi_{\varphi}\left(\left(b_{n}^{(p)}\right)^{*} b_{n}^{(p)}\right)\right\}=\sup _{n} s_{c}\left\{\pi_{\varphi}\left(b_{n}^{(p)}\left(b_{n}^{(p)}\right)^{*}\right)\right\}=1
$$

where $\pi_{\varphi}$ is the cyclic representation of $\mathfrak{U}^{G}$ associated with $\varphi$ and $s_{c}$ denotes the central support in $\pi_{\varphi}\left(\mathfrak{A}^{G}\right)^{\prime \prime}$.

Our main result is summarized in the following theorem:

Theorem 1. Under Assumption A, the following holds:

(1) If $\varphi$ is an extremal $\varrho_{t}$-KMS state of $\mathfrak{H}^{G}$ satisfying Assumption $B$, then $\varphi^{-}=\varphi \circ \varepsilon_{1}$ is an extremal G-invariant $\varrho_{t} \cdot \alpha_{t}$-KMS state of $\mathfrak{A}$ for a one-parameter subgroup $\alpha_{t}$ of $G$.

(2) If $\varphi^{\wedge}$ is a $\varrho_{t} \cdot \alpha_{t}$-KMS state of $\mathfrak{A}$ for a one-parameter subgroup $\alpha_{t}$ of $G$, then the restriction $\varphi$ of $\varphi^{\wedge}$ to $\mathfrak{U}^{G}$ is a $\varrho_{t}$-KMS state of $\mathfrak{Q}^{G}$ satisfying Assumption $B$. If $\varphi^{\wedge}$ is primary, then $\varphi$ is an extremal $\varrho_{t}-\mathrm{KMS}$ state of $\mathfrak{H}^{\mathrm{G}}$. If $\varphi^{\wedge}$ is $G$-invariant, then $\varphi^{\wedge}=\varphi \circ \varepsilon_{1}$. If $\varphi^{\wedge}$ is extremal among $G$-invariant $\varrho_{t} \cdot \alpha_{t}$-KMS states of $\mathfrak{H}$, then $\varphi$ is an extremal $\varrho_{t}-\mathrm{KMS}$ state of $\mathfrak{H}^{G}$.

(3) Extremal $\varrho_{t}-\mathrm{KMS}$ states of $\mathfrak{U}^{G}$ satisfying Assumption $B$ are in one-to-one correspondence with extremal $G$-invariant $\varrho_{t} \cdot \alpha_{t}$-KMS states of $\mathfrak{U}$ with $\alpha$ varying over one-parameter subgroups of $G$, where the correspondence is through restriction and $G$-invariant extension.

(4) For any extremal $\varrho_{t}$-KMS state $\varphi$ of $\mathfrak{U}^{G}$ satisfying Assumption $B$, there exists an extremal $\varrho_{t} \circ \alpha_{t}$-KMS state $\varphi^{\wedge}$ for some one-parameter subgroup $\alpha_{t}$ of $G$ such that its restriction to $\mathfrak{A}^{G}$ is $\varphi$. If $\varphi^{\wedge} \circ g_{1} \neq \varphi^{\wedge} \circ g_{2}$ for $g_{1}$ and $g_{2} \in G$, then the cyclic representations associated with $\varphi^{\wedge} \circ g_{1}$ and $\varphi^{\wedge} \circ g_{2}$ are disjoint. The central decomposition of the G-invariant extension $\varphi^{-}$of $\varphi$ to $\mathfrak{A}$ is given by

$$
\varphi^{-}=\int \varphi^{\wedge} \circ g d g \text {. }
$$

Remark 1. It is well-known that a $\mathrm{KMS}$ state $\varphi$ of a $C^{*}$-algebra $\mathfrak{A}$ relative to a continuous one-parameter group of *automorphisms is extremal if and only if it is primary [i.e. the center of the weak closure $\pi(\mathfrak{H})^{\prime \prime}$ of the cyclic representation $\pi_{\varphi}$ associated with $\varphi$ is trivial].

Remark 2. An example of the case satisfying the Assumption A is given by quantum spin lattice system, where we may take (mathematically) $G$ to be the (spin) rotation 
group around the $z$-axis. Let $i_{n}$ be lattice sites tending to infinity as $n \rightarrow \infty, \Delta$ consist of just one character $e^{i \theta}$ and $b_{n}$ (for this character) be the spin operator $\sigma_{x}+i \sigma_{y}$ at $i_{n}$-th lattice site. Then Assumption A is clearly satisfied and hence the conclusion of Theorem 1 holds.

Remark 3. The items (c) and (d) in Assumption A obviously follow from the following stronger but simpler assumption: $\lim _{n}\left\|\left[b_{n}^{(p)}, x\right]\right\|=0$ for all $x \in \mathfrak{A}$. However, we want to avoid the assumption of such a kind that amounts to assuming asymptotic commutativity of fields at large space distance. In the stated form of Assumption A, it follows from asymptotic commutativity of observables with fields. The assumption (e) along with other assumptions are satisfied in asymptotically abelian system, i.e. if there exists a sequence of automorphisms $\tau_{n}$ of $\mathfrak{A}$ commuting with $\varrho_{t}$ and $G$ such that $\lim _{n}\left\|\left[\tau_{n}(a), b\right]\right\|=0$ for any $a \in \mathfrak{H}^{G}$ and $b \in \mathfrak{A}$ and if there exists $b^{(p)} \in \varepsilon_{p}(\mathfrak{Q})$ for $p \in \Delta$ such that $b^{(p)}\left(b^{(p)}\right)^{*}+\left(b^{(p)}\right)^{*} b^{(p)} \geqq 1$.

Remark 4. If $\varphi$ is an extremal $\varrho_{t}$-KMS state, Assumption B is equivalent to the requirement that $\pi_{\varphi}\left(\left(b_{n}^{(p)}\right)^{*} b_{n}^{(p)}\right)$ and $\pi_{\varphi}\left(b_{m}^{(p)}\left(b_{m}^{(p)}\right)^{*}\right)$ are non-zero for some $n$ and $m$. [It can be weakened to the requirement that $\pi_{\varphi}\left(a^{*} a\right) \neq 0$ for some $a \in \varepsilon_{p}(\mathfrak{H})$ for each $p \in \Delta \cup \Delta^{-1}$.] The situation where Assumption B is violated is discussed in Section 9. It leads to a one-sided spectrum (temperature 0 ) for the representation of $G$ associated with $\varphi^{-}$, when $G$ is one-dimensional torus.

Remark 5. $\varphi^{\wedge}$ of Theorem 1(4) might be non-invariant under time translation, possibly periodic or aperiodic.

The situation for a general $\varrho_{t}$-KMS state of $\mathfrak{A}^{G}$ is contained in the following theorem:

Theorem 2. Under Assumption A, the following holds for any G-invariant state $\varphi^{-}$ of $\mathfrak{A}$, whose restriction to $\mathfrak{P}^{G}$ is a $\varrho_{t}$-KMS state $\varphi$ of $\mathfrak{P}^{G}$ satisfying Assumption $B$.

(1) For $M=\pi_{\bar{\varphi}}(\mathfrak{A})^{\prime \prime}$ and $M^{G}=\pi_{\bar{\varphi}}\left(\mathfrak{Q}^{G}\right)^{\prime \prime}$, the center of $M^{G}$ is contained in the center of $M$.

(2) The cyclic vector $\Omega$ associated with $\varphi^{-}$is separating for $M$.

(3) If $\varphi$ is an extremal $\varrho_{t}$-KMS state, then $M^{G}$ is a factor.

Remark 6. The central decomposition of $\varphi$ is so-to-speak the diagonalization of the center of $M^{G}$ and is known to coincide with a decomposition into extremal $\varrho_{t}$-KMS states. The central decomposition

$$
\varphi=\int \xi d \mu(\xi)
$$

of $\varphi$ into extremal $\varrho_{t}$-KMS states $\xi$ of $\mathfrak{A}^{G}$ induce a decomposition of $\varphi^{-}=\varphi{ }^{\circ} \varepsilon_{1}$ :

$$
\varphi^{-}=\int \xi^{-} d \mu(\xi), \xi^{-}=\xi_{\circ} \varepsilon_{1},
$$

which is so-to-speak a decomposition diagonalizing the center of $M^{G}$. By Theorem 2(1), this will be a part of the central decomposition of $\varphi^{-}$, which can be achieved by combining the central decompositions of $\xi^{-}$with the above decomposition. 


\section{§3. Preliminaries}

Theorem 1 (1) contains three assertions: $\varphi^{-}$is $G$-invariant, $\varphi^{-}$is a $\varrho_{t} \cdot \alpha_{t}$-KMS state of $\mathfrak{A}$ for some $\alpha$ and $\varphi^{-}$is extremal among $G$-invariant $\varrho_{t} \cdot \alpha_{t}-$ KMS states. The first assertion is immediate from the definition $\varphi^{-}=\varphi \circ \varepsilon_{1}$ due to $\varepsilon_{1} \circ g=\varepsilon_{1}$ for all $g \in G$. The second assertion is most important part which occupies the main portion of Section 5. The third assertion is proved without Assumption A. We start with the proof of the third assertion, which obviously follows from the following two Lemmas, where

$$
M=\pi_{\bar{\phi}}(\mathfrak{A})^{\prime \prime}, M^{G}=\pi_{\bar{\phi}}\left(\mathfrak{Q}^{G}\right)^{\prime \prime} .
$$

Lemma 1. A G-invariant $\varrho_{t} \cdot \alpha_{t}-\mathrm{KMS}$ state $\varphi^{-}$of $\mathfrak{A}$ is extremal among such states if and only if $M^{G} \cap M^{\prime}$ is trivial.

Lemma 2. If $\varphi^{-}$is a G-invariant $\varrho_{t} \cdot \alpha_{t}-\mathrm{KMS}$ state of $\mathfrak{A}$ and if the restriction of $\varphi^{-}$ to $\mathfrak{A}^{G}$ is an extremal $\varrho_{t}-\mathrm{KMS}$ state, then $M^{G} \cap M^{\prime}$ is trivial.

Before presenting the proof of these Lemmas, we introduce a few notations connected with a $G$-invariant state $\varphi^{-}$of $\mathfrak{A}$ and the associated cyclic representation $\pi_{\bar{\varphi}}$. By $G$-invariance of $\varphi^{-}$, there exists a continuous unitary representation $U(g)$, $g \in G$, of $G$ such that

$$
U(g) \pi_{\bar{\varphi}}(a) \Omega=\pi_{\bar{\varphi}}(g a) \Omega, a \in \mathfrak{A} .
$$

The continuous extension of $g \in G$ to $M=\pi_{\bar{\varphi}}(\mathfrak{Q})^{\prime \prime}$ is defined by

$$
g^{-}(x)=U(g) x U(g)^{*}, x \in M .
$$

The continuous extension of $\varepsilon_{p}, p \in G^{\wedge}$, to $M$ is defined by

$$
\varepsilon_{p}^{-}(x)=\int p(g)^{*} g^{-}(x) d g, x \in M, p \in G^{\wedge} \text {. }
$$

Since $\varepsilon_{p}^{-}$is continuous, $\pi_{\bar{\phi}}\left(\varepsilon_{p}(\mathfrak{R})\right)$ is dense in $\varepsilon_{p}^{-}(M)$. In particular the set of $G$ invariant elements of $M$ is given by $\varepsilon^{-}(M)=\pi_{\bar{\varphi}}\left(\mathfrak{A}^{G}\right)^{\prime \prime}=M^{G}$. More generally, $\varepsilon_{p}^{-}(M)$ is the set of all $x \in M$ satisfying $g(x)=p(g) x$ for all $g \in G$. Hence $\varepsilon_{p}^{-}(M) \varepsilon_{q}^{-}(M) C$ $\varepsilon_{p q}^{-}(M)$.

Proof of Lemma 1. Let $e$ be a projection operator in $M^{G} \cap M^{\prime}$. Since $e$ is in the center of $M$,

$$
\varphi^{-}=\varphi^{-}+\varphi^{-}{ }_{1-e}
$$

and both $\varphi_{e}^{-}$and $\varphi_{1-e}^{-}$are $\varrho_{t} \cdot \alpha_{t}$-KMS states, where $\varphi_{E}^{-}$denotes the normal linear functional on $M$ defined by a vector $e \Omega$. $\left(\varphi^{-}{ }_{1}=\varphi^{-}\right.$and $\varphi^{-}{ }_{0}=0$.) Since $e$ is invariant under $g^{-}$for all $g \in G$, both $\varphi_{e}^{-}$and $\varphi^{-}{ }_{1-e}$ are $G$-invariant. If $\varphi_{e}^{-}=k \varphi^{-}$ for a number $k$, then

$$
k\left(\pi_{\varphi}\left(x_{1}\right) \Omega, \pi_{\varphi}\left(x_{2}\right) \Omega\right)=\left(\pi_{\varphi}\left(x_{1}\right) \Omega, e \pi_{\varphi}\left(x_{2}\right) \Omega\right)
$$

due to $e \in M^{\prime}$, and hence $e=k \cdot 1$ due to the cyclicity of $\Omega$. Hence $e=1$ or 0 . This implies that $M^{G} \cap M^{\prime}$ is trivial if $\varphi^{-}$is extremal among $G$-invariant $\varrho_{t} \cdot \alpha_{t}$-KMS states.

To prove the converse assertion, we assume that

$$
\varphi^{-}=\varphi_{a}^{-}+\varphi_{b}^{-}
$$


where $\varphi_{a}^{-}$and $\varphi_{b}^{-}$are both $G$-invariant $\varrho_{t} \cdot \alpha_{t}$-KMS positive linear functionals. Since $\varphi^{-}{ }_{a} \leqq \varphi^{-}$, there exists an operator $a \in M^{\prime}$ such that $0 \leqq a \leqq 1$ and $\varphi_{a}^{-}$is given as the vector linear functional by the vector $a \Omega$. The assumption that $\varphi^{-}$and $\varphi^{-}$ are both $\varrho_{t} \cdot \alpha_{t}$-KMS positive linear functionals implies that $a$ is in the center of $M$. The $G$-invariance of $\varphi_{a}^{-}$implies that $g^{-}(a)=a$ for all $g \in G$. Hence $a$ is in $M^{G} \cap M^{\prime}$. If $M^{G} \cap M^{\prime}$ is trivial, then $a$ must be a multiple of the identity and hence $\varphi^{-}$is a multiple of $\varphi^{-}$. Therefore, $\varphi^{-}$is an extremal $G$-invariant $\varrho_{t} \cdot \alpha_{t}-\mathrm{KMS}$ state if $M^{G} \cap M^{\prime}$ is trivial.

Q.E.D.

Proof of Lemma 2. If the restriction of $\varphi^{-}$to $\mathfrak{Q}^{G}$ is an extremal KMS-state, then the restriction of $M^{G}=\pi\left(\mathfrak{A}^{G}\right)^{\prime \prime}$ to the cyclic subspace $\mathfrak{S}_{1} \equiv \overline{M^{G} \Omega}$ is a factor. Since $\Omega$ is $M$-cyclic, $\left(M \cap M^{\prime}\right)$-support of $\Omega$ is 1 , namely $\Omega$ is separating for $M \cap M^{\prime}$ and hence for its subalgebra $M^{G} \cap M^{\prime}$. Since $M^{G} \cap M^{\prime}$ is contained in the center of $M^{G}$, the restriction of $M^{G} \cap M^{\prime}$ to $\mathfrak{H}_{1}$ is trivial. Since $\Omega \in \mathfrak{H}_{1}$ is separating for $M^{G} \cap M^{\prime}$, this implies that $M^{G} \cap M^{\prime}$ is trivial.

Q.E.D.

\section{§ 4. Proof of Theorem 2}

We first prove a technical Lemma, which is actually an implicit form of $\varrho_{t} \circ \alpha_{t}$-KMS condition for $\varphi^{-}$.

Lemma 3. Let $\mathscr{F}_{1}$ be the algebra of bounded continuous functions on the strip region

$$
T=\{z \in C ; 0 \leqq \operatorname{Im} z \leqq 1\}
$$

which are holomorphic in the interior of $T$ and $\mathscr{F}$ be the algebra of $\left(M \cap M^{\prime}\right)$-valued entire functions which are uniformly bounded on $T$. For each $q \in G^{\wedge}$, there exist $F_{q}$ and $G_{q}$ in $\mathscr{F}$ such that support projections $s\left(F_{q}(t)\right)$ and $s\left(G_{q}(t)\right)$ satisfy

$$
\lim _{\varepsilon \rightarrow+0} \sup _{|t|<\varepsilon} s\left(F_{q}(t)\right)=\lim _{\varepsilon \rightarrow+0} \sup _{|t|<\varepsilon} s\left(G_{q}(t)\right)=1
$$

and for any $x \in \varepsilon_{q}^{-}(M)$ and $b \in \varepsilon_{q}(\mathfrak{l})$, there exists an $F_{x, b} \in \mathscr{F}_{1}$ satisfying

$$
\left.\begin{array}{rl}
F_{x, b}(t) & =\left(\Omega, x^{*} \pi_{\bar{\phi}}\left(\varrho_{t}(b)\right) F_{q}(t) \Omega\right), \\
F_{x, b}(t+i) & =\left(\Omega, \pi_{\bar{\phi}}\left(\varrho_{t}(b)\right) x^{*} G_{q}(t) \Omega\right),
\end{array}\right\} .
$$

For each $q_{1}, q_{2} \in G^{\wedge}$, it is possible to choose $F$ 's and $G$ 's so that $F_{q_{1}} F_{q_{2}}=F_{q_{1} q_{2}}$ and $G_{q_{1}} G_{q_{2}}=G_{q_{1} q_{2}}$.

Proof. For given $b_{n}^{(p)}$ in Assumption A, $\varrho_{t}\left(b_{n}^{(p)}\right)$ satisfies all conditions for $b_{n}^{(p)}$ and hence

$$
b_{n}^{\sim(p)} \equiv \int_{-\infty}^{\infty}(\pi \varepsilon)^{-1 / 2} \exp \left(-t^{2} / \varepsilon\right) \varrho_{t}\left(b_{n}^{(p)}\right) d t
$$

for $\varepsilon>0$ also satisfies all conditions of Assumption A except for possibly (b). Since $b_{n}^{\sim(p)} \rightarrow b_{n}^{(p)}$ in norm as $\varepsilon \rightarrow+0, \lambda b_{n}^{\sim(p)}$ for small $\varepsilon$ and large $\lambda$ will satisfy all conditions for $b_{n}^{(p)}$. In addition, $\varrho_{t}\left(b_{n}^{\sim(p)}\right)$ is an entire function of $t$. In the following, we assume that this replacement has been made in Assumption A. We then have $\varrho_{z}\left(b_{n}^{(p)}\right)$ for all complex $z$ and Assumptions A(a), (c), (d), and (e) are satisfied even when $t$ is replaced by $z$. 
Since $\left(b_{n}^{(p)}\right)^{*}$ satisfies all conditions for $b_{n}^{(q)}, q=p^{-1}$, we assume that $p^{-1} \in \Delta$ if $p \in \Delta$ and use $\left(b_{n}^{(p)}\right)^{*}$ for $b_{n}^{\left(p^{-1}\right)}$ if necessary. [This will be used in particular in the proof of Theorem 2(1).]

By Assumption A and compactness argument, there exists a subnet $n(v)$ such that

$$
\left.\begin{array}{l}
w-\lim \pi_{\varphi} \hat{\varphi}\left(b_{n(v)}^{(p)} \varrho_{t}\left(b_{n(v)}^{(p)}\right)^{*}\right)=f_{p}(t), \\
w-\lim \pi_{\varphi}\left(\varrho_{t}\left(b_{n(v)}^{(p)}\right) * b_{n(v)}^{(p)}\right)=g_{p}(t),
\end{array}\right\}
$$

exist (simultaneously for a finite number of $p$ 's if necessary). By Assumption A(d), both $f_{p}(t)$ and $g_{p}(t)$ belong to $M^{G} \cap M^{\prime}$. By our choice of $b_{n}^{(p)}$ and Assumption A(e), both $f_{p}(t)$ and $g_{p}(t)$ are entire functions of $t$. By Assumption $\mathrm{A}(\mathrm{b})$, we have $f_{p}(0)+$ $g_{p}(0) \geqq 1$.

Let $a, b \in \varepsilon_{q}(\mathfrak{U}), q=p_{1} \ldots p_{n}, p_{j} \in \Delta$. By $\varrho_{t}-\mathrm{KMS}$ condition for $\varphi$, there exists $F_{\left\{v_{j}\right\}} \in \mathscr{F}_{1}$ such that

$$
\begin{aligned}
F_{\left\{v_{j}\right\}}(t) & =\varphi^{-}\left(\left\{a^{*} b_{n\left(v_{1}\right)}^{\left(p_{1}\right)} \ldots b_{n\left(v_{n}\right)}^{\left(p_{n}\right)}\right\} \varrho_{t}\left\{b^{*} b_{n\left(v_{1}\right)}^{\left(p_{1}\right)} \ldots b_{n\left(v_{n}\right)}^{\left(p_{n}\right)}\right\}^{*}\right), \\
F_{\left\{v_{j}\right\}}(t+i) & =\varphi^{-}\left(\varrho_{t}\left\{b^{*} b_{n\left(v_{1}\right)}^{\left(p_{1}\right)} \ldots b_{n\left(v_{n}\right)}^{\left(p_{n}\right)}\right\}^{*}\left\{a^{*} b_{n\left(v_{1}\right)}^{\left(p_{1}\right)} \ldots b_{n\left(v_{n}\right)}^{\left(p_{n}\right)}\right\}\right),
\end{aligned}
$$

for all real $t$. By taking limits successively for nets $n\left(v_{n}\right), \ldots, n\left(v_{1}\right)$ and using Assumption $\mathrm{A}(\mathrm{c})$, we obtain

$$
\begin{aligned}
\underset{v_{1}}{\lim } \ldots \lim _{v_{n}} F_{\left\{v_{j}\right\}}(t) & =\left(\Omega, \pi_{\bar{\varphi}}\left(a^{*} \varrho_{t}(b)\right) \prod_{j=1}^{n} f_{p_{j}}(t) \Omega\right), \\
\lim _{v_{1}} \ldots \lim _{v_{n}} F_{\left\{v_{j}\right\}}(t+i) & =\left(\Omega, \pi_{\bar{\varphi}}\left(\varrho_{t}(b) a^{*}\right) \prod_{j=1}^{n} g_{p_{j}}(t) \Omega\right) .
\end{aligned}
$$

By Assumption A(e), the limit is uniform over a compact set of $t$ and the net is uniformly bounded by Assumption A(a). By a standard argument, we obtain [as the limit of $\left.F_{\left\{v_{j}\right\}}(z)\right]$ the desired $F_{a, b} \in \mathscr{F}$ satisfying (4.2) for $x=\pi_{\bar{\varphi}}(a)$ with

$$
F_{q}(z)=\prod_{j=1}^{n} f_{p_{j}}(z), G_{q}(z)=\prod_{j=1}^{n} g_{p_{j}}(z) \text {. }
$$

It is obvious from (4.4) that the decompositions $q_{1}=\Pi p_{j}, q_{2}=\Pi p_{j}^{\prime}, q_{1} q_{2}=$ $\left(\Pi p_{j^{\prime}}^{\prime}\right)\left(\Pi p_{j}\right)$ will yield $F^{\prime}$ 's and $G$ 's satisfying $F_{q_{1}} F_{q_{2}}=F_{q_{1} q_{2}}, G_{q_{1}} G_{q_{2}}=G_{q_{1} q_{2}}$.

As a net $\pi_{\bar{\varphi}}\left(a_{\alpha}\right)$ tends $*_{\text {-strongly to } x \in \varepsilon_{q}^{-}}^{-}(M),(4.2)$ approaches to

$$
\left.\begin{array}{rl}
F_{x, b}(t) & \equiv\left(\Omega, x^{*} \pi_{\bar{\varphi}}\left(\varrho_{t}(b)\right) F_{q}(t) \Omega\right) \\
F_{x, b}(t+i) & \equiv\left(\Omega, \pi_{\bar{\varphi}}\left(\varrho_{t}(b)\right) G_{q}(t) x^{*} \Omega\right)
\end{array}\right\}
$$

uniformly over $t$, and hence $F_{x_{\alpha}, b}(z), x_{\alpha}=\pi_{\bar{\varphi}}\left(a_{\alpha}\right)$, tends to $F_{x, b}(z)$ in $\mathscr{F}_{1}$ satisfying (4.5). [Note that $G_{q}(t) \in M \cap M^{\prime}$.] Let $q=p \in \Delta, e$ be a projection operator in $M^{G} \cap M^{\prime}$ such that $e f_{p}(t)$ is zero in a neighbourhood of $t=0, b=b_{m}^{(p)}$ and $x=e \pi_{\bar{\varphi}}(b)$. Then $F_{x, b}(z)$ is identically 0 . Hence

$$
\|\left\{e g_{p}(0) \pi_{\bar{\varphi}}\left(b b^{*}\right)^{1 / 2} \Omega \|^{2}=F_{x, b}(i)=0 .\right.
$$

Since $\Omega$ is separating for the restriction of $M^{G}$ to the weak closure of $M^{G} \Omega$ due to $\varrho_{t}$-KMS condition for $\varphi$, we obtain

$\left[e g_{p}(0)\right]_{1}\left[\pi_{\bar{\varphi}}\left(b_{m}^{(p)}\left(b_{m}^{(p)}\right)^{*}\right)\right]_{1}=0$ 
for all $m$, where $[y]_{1}$ denotes the restriction of $y \in M^{G}$ to the weak closure of $M^{G} \Omega$. Hence $\left[e g_{p}(0)\right]_{1}=0$ due to Assumption B. Since $\Omega$ is separating for $M^{\prime} \supset M^{G} \cap M^{\prime}$, we have $e g_{p}(0)=0$. Hence $e\left(f_{p}(0)+g_{p}(0)\right)=0$. Since $f_{p}(0)+g_{p}(0) \geqq 1$, we obtain $e=0$. This proves

$$
\lim _{\varepsilon \rightarrow+0} \sup _{|t|<\varepsilon} s\left(F_{q}(t)\right)=1
$$

for $q=p \in \Delta$. The same proof works for $G_{q}(t)$.

The proof will be completed if we show that (4.6) for $q_{1}$ and $q_{2}$ imply (4.6) for $F_{q_{1} q_{2}}(t)=F_{q_{1}}(t) F_{q_{2}}(t)$.

Suppose that there exists a projection $e$ in $M^{G} \cap M^{\prime}$ such that $e F_{q_{1} q_{2}}(t)=0$ for all $|t| \leqq \varepsilon$ for some $\varepsilon>0$. Let $e_{j}(t)=e\left\{1-s\left(F_{q_{j}}(t)\right)\right\}$. If $e-e_{1}(t) \neq 0$ for $t=t_{0}$, then there exists a non-zero $e_{0} \leqq e-e_{1}(t)$ such that $e_{0} \leqq e-e_{1}(t)$ for all $t$ in some neighbourhood of $t=t_{0}$ due to the norm-continuity of $F_{q_{1}}(t)$. Hence $e_{0} F_{q_{2}}(t)=0$ for the same neighbourhood and hence $e_{0} F_{q_{2}}(t)=0$ for all $t$ by the analyticity. This contradicts with (4.6) for $q=q_{2}$. Hence $e=e_{1}(t) \leqq 1-s\left(F_{q_{1}}(t)\right)$. This implies $e F_{q_{1}}(t)=0$ and hence $e=0$ by (4.6) for $q=q_{1}$. Hence (4.6) holds for $q=q_{1} q_{2}$. The same proof holds for $G$ 's.

Q.E.D.

Proof of Theorem 2(1). It is enough to prove that $x \in M^{G} \cap\left(M^{G}\right)^{\prime}$ commutes with $\pi_{\varphi}(y) \in \pi_{\varphi}\left(\varepsilon_{p}(\mathfrak{H})\right)$ for all $p \in \Delta$. Let $a \in \varepsilon_{p q}(\mathfrak{H}), b \in \varepsilon_{q}(\mathfrak{H})$. Since $y\left(b_{n(v)}^{(p)}\right) * \in \mathfrak{H}^{G}$ and $x \in\left(M^{G}\right)^{\prime}$, we have

$$
\begin{aligned}
& \left(\Omega, \pi_{\bar{\phi}}(a)^{*} x \pi_{\bar{\varphi}}\left\{y\left(b_{n(v)}^{(p)} * \varrho_{t}\left(b_{n(v)}^{(p)} b\right)\right\} F_{p q}(t) \Omega\right)\right. \\
& =\left(\Omega, \pi_{\bar{\varphi}}\left(a^{*} y\left(b_{n(v)}^{(p)}\right) * x \pi_{\bar{\varphi}}\left\{\varrho_{t}\left(b_{n(v)}^{(p)} b\right)\right\} F_{p q}(t) \Omega\right) .\right.
\end{aligned}
$$

By Lemma 3, the analytic continuation in the variable $t$ by an amount $i$ yields

$$
\left(\Omega, \pi_{\bar{\phi}}\left\{\varrho_{t}\left(b_{n(v)}^{(p)} b\right)\right\} \pi_{\bar{\varphi}}\left(a^{*} y\left(b_{n(v)}^{(p)}\right) *\right) x G_{p q}(t) \Omega\right) .
$$

By taking $v$-limit and using Assumption $\mathrm{A}(\mathrm{c})$ and (e), we obtain an $F \in \mathscr{F}_{1}$ such that

$$
\begin{aligned}
F(t) & =\left(\Omega, \pi_{\bar{\phi}}(a)^{*} x \pi_{\bar{\varphi}}\left\{y \varrho_{t}(b)\right\} F_{\left(p^{-1}\right)}(t) \dot{F}_{p q}(t) \Omega\right), \\
F(t+i) & =\left(\Omega, \pi_{\bar{\varphi}}\left\{\varrho_{t}(b) a^{*} y\right\} G_{\left(p^{-1}\right)}(t) x G_{p q}(t) \Omega\right) .
\end{aligned}
$$

By Lemma 3, we obtain by our convention $F_{\left(p^{-1}\right)} F_{p q}=F_{q}$ and $G_{\left(p^{-1}\right)} G_{p q}=G_{q}$, the following equality

$$
\left(\Omega, \pi_{\bar{\varphi}}\left(a^{*}\right)\left[x, \pi_{\bar{\varphi}}(y)\right] \pi_{\bar{\varphi}}\left(\varrho_{t}(b)\right) F_{q}(t) \Omega\right)=0,
$$

for all real $t$. Since $b$ is an arbitrary element of $\varepsilon_{q}(\mathfrak{A})$, which is $\varrho_{t}$-invariant as a set we may replace $\varrho_{t}(b)$ by $b$ for each $t$.

Hence

$$
\left(\pi_{\bar{\varphi}}(a) \Omega,\left[x, \pi_{\bar{\varphi}}(y)\right] F_{q}(t) \pi_{\bar{\varphi}}(b) \Omega\right)=0 .
$$

The Equation (4.7) holds for $a \in \varepsilon_{q_{1}}(\mathfrak{l})$ and $b \in \varepsilon_{q_{2}}(\mathfrak{l})$ by $G$-invariance of $\varphi^{-}$if $q_{1} q_{2}^{-1} \neq p$ and by the above argument if $q_{1} q_{2}^{-1}=p$. Since $\pi_{\bar{\varphi}}\left(\varepsilon_{r}(\mathfrak{R})\right) \Omega, r \in G^{\wedge}$, is total, we obtain $\left[x, \pi_{\bar{\varphi}}(y)\right] F_{q}(t)=0$. By (4.6), we obtain $\left[x, \pi_{\bar{\varphi}}(y)\right]=0$.

Q.E.D. 
Remark. Assumption A(c) implies

$$
w-\lim _{n}\left[x, \pi_{\bar{\varphi}}\left(b_{n}^{(p)}\right)^{*}\right]=0
$$

for all $x \in M^{G}$. If (4.8) holds in the *-strong operator topology (instead of the weak operator topology), then Theorem 2(1) has the following easy proof: For $x \in M^{G} \cap$ $\left(M^{G}\right)^{\prime}$, we have

$$
\begin{aligned}
& {\left[x, y \pi_{\bar{\varphi}}\left(b_{n}^{(p)}\right)^{*}\right] \pi_{\bar{\varphi}}\left(b_{n}^{(p)}\right)=0,} \\
& \pi_{\bar{\varphi}}\left(b_{n}^{(p)}\right)\left[x, y \pi_{\bar{\varphi}}\left(b_{n}^{(p)}\right)^{*}\right]=0,
\end{aligned}
$$

for any $y \in \pi_{\bar{\varphi}}\left(\varepsilon_{p}(\mathfrak{A})\right)$. By taking limit, we obtain $[x, y] f_{p}(0)=[x, y] g_{p}(0)=0$. Hence $f_{p}(0)+g_{p}(0) \geqq 1$ implies $[x, y]=0$.

Proof of Theorem 2(2). Let $\Omega$ be the cyclic vector associated with $\varphi^{-}$. The $\left(M \cap M^{\prime}\right)$ support of $\Omega$ is 1 because of the cyclicity of $\Omega$. By Theorem 2(1), the center of $M^{G}$ is contained in $M \cap M^{\prime}$ and hence $\left(M^{G} \cap\left(M^{G}\right)^{\prime}\right)$-support of $\Omega$ is also 1 . This implies that $M^{G}$ is isomorphic to its restriction to the cyclic subspace

$$
\mathfrak{H}_{1}=\text { the closure of } \pi_{\bar{\varphi}}\left(\mathfrak{U}^{G}\right) \Omega \text {. }
$$

Since the normal extension of a KMS-state to the weak closure of the associated cyclic representation is known to be faithful, $\Omega$ must be separating for the restriction of $M^{G}$ to $\mathfrak{H}_{1}$, hence for $M^{G}$ itself.

Let $x \Omega=0$ and $x \in M$. We have to prove $x=0$. Since $g^{-}(x) \Omega=U(g) x \Omega=0$, $\varepsilon_{p}^{-}(x) \Omega=0$ for all $p \in G^{\wedge}$. Hence

$$
\varepsilon_{p}^{-}(x)^{*} \varepsilon_{p}^{-}(x) \Omega=0
$$

for $\varepsilon_{p}^{-}(x)^{*} \varepsilon_{p}^{-}(x) \in M^{G}$. By (2), we obtain $\varepsilon_{p}^{-}(x)^{*} \varepsilon_{p}^{-}(x)=0$ and hence $\varepsilon_{p}^{-}(x)=0$ for all $p \in G^{\wedge}$.

For $a \in \varepsilon_{p}^{-}(M)$, we have

$$
U(g) a \Omega=U(g) a U(g) * \Omega=p(g) a \Omega .
$$

Hence for $a \in \varepsilon_{p}^{-}(M)$ and $b \in \varepsilon_{q}^{-}(M)$, we have

$$
\begin{aligned}
(a \Omega, x b \Omega) & =\left(a \Omega, U(g) x U(g)^{*} r(g)^{*} b \Omega\right) \\
& =\left(a \Omega, \varepsilon_{r}^{-}(x) b \Omega\right)=0
\end{aligned}
$$

where $r=p q^{-1}$. Since $\left\{\varepsilon_{p}^{-}(M) \Omega ; p \in G^{\wedge}\right\}$ is total, we obtain $x=0$.

Proof of Theorem 2(3). A KMS state is extremal if and only if it is primary. Hence the restriction of $M^{G}$ to $\mathfrak{H}_{1}$, must be a factor if the restriction of $\varphi^{-}$to $\mathfrak{A}^{G}$ is an extremal $\varrho_{t}$-KMS state. By the above proof of (2), $M^{G}$ itself is a factor.

\section{§. Proof of Theorem 1 (1)}

By Theorem 2(3), $M^{G}$ is a factor. In the following proof, we use Assumption A and $\mathrm{B}$ only through the property that $M^{G}$ is a factor, except Lemma 5 where we use Assumption A. We will not use Lemma 5 until the very end of Proof of Theorem 1(1) where it is used in a trivial way. 
Lemma 4. If $M^{G}$ is a factor, then the set $H$ of $p \in G^{\wedge}$ such that $\varepsilon_{p}^{-}(M) \neq 0$ is a subgroup of $G^{\wedge}$.

Proof. We know that $M^{G}=\varepsilon^{-}{ }_{1}(M) \neq 0$. Let $x \in \varepsilon^{-}(M)$ and $x \neq 0$. Then $x^{*} \in \varepsilon_{q}^{-}(M)$ for $q=p^{-1}$. Hence $\varepsilon_{p}^{-}(M) \neq 0$ and $\varepsilon_{q}^{-}(M) \neq 0$ are equivalent if $p q=1$. Next, let $x_{i} \in \varepsilon_{p_{i}}^{-}(M)$ and $x_{i} \neq 0, i=1,2$. Then $x_{i}^{*} x_{i} \in M^{G}$ and $x_{i} x_{i}^{*} \in M^{G}$. Since $M^{G}$ is a factor, there exists $u \in M^{G}$ such that $\left(x_{1}^{*} x_{1}\right) u\left(x_{2} x_{2}^{*}\right) \neq 0$. Then $x_{1} u x_{2} \neq 0$ and $x_{1} u x_{2} \in \varepsilon_{q}^{-}(M)$ for $q=p_{1} p_{2}$.

Q.E.D.

Lemma 5. $H=G^{\wedge}$.

Proof. Assumption A implies $\pi_{\varphi}\left(b_{n}^{(p)}\right) \neq 0$ for any $\varphi^{-}$and $p \in \Delta$.

Lemma 6. If $M^{G}$ is a factor and $\varepsilon_{p}^{-}(M) \neq 0$, then there exists either isometric or co-isometric operator u in $\varepsilon_{p}^{-}(M)$.

Proof. Let $x \in \varepsilon_{p}^{-}(M), x \neq 0$. Then $x^{*} x \in M^{G}$ and hence $|x| \in M^{G}$. The polar decomposition $x=u|x|$ gives a partially isometric operator $u=\lim _{\varepsilon \rightarrow+0} x(\varepsilon+|x|)^{-1} \in \varepsilon_{p}^{-}(M)$.

Since $x \neq 0, u$ is non-zero. Let $u^{*} u=e, u u^{*}=e^{\prime}$. They are projection operators in $M^{G}$. The map $x \in M_{e}^{G} \rightarrow x^{\prime} \equiv u x u^{*} \in M_{e^{\prime}}^{G}$ is a *-isomorphism of $M_{e}^{G}$ onto $M_{e^{\prime}}^{G}$.

First we consider the case where $M^{G}$ is properly infinite. Then there exist two sets of projections $\left\{e_{\alpha}^{(1)}\right\}$ and $\left\{e_{\alpha}^{(2)}\right\}$ with the same index set such that $e_{\alpha}^{(j)} \in M^{G}$, $e_{\alpha}^{(j)} e_{\beta}^{(j)}=0$ for $\alpha \neq \beta, \sum e_{\alpha}^{(j)}=1$, all $e_{\alpha}^{(1)}$ are equivalent to $e$ and all $e_{\alpha}^{(2)}$ are equivalent to $\ddot{e}^{\prime}$ [i.e. there exist $u_{\alpha}^{(j)} \in M^{G}$ satisfying $\left\{u_{\alpha}^{(j)}\right\}^{*} u_{\alpha}^{(j)}=e_{\alpha}^{(j)}, u_{\alpha}^{(1)}\left\{u_{\alpha}^{(1)}\right\}^{*}=e$, and $\left.u_{\alpha}^{(2)}\left\{u_{\alpha}^{(2)}\right\}^{*}=e^{\prime}\right]$ where $j=1,2$. Then

$$
U=\sum_{\alpha}\left\{u_{\alpha}^{(2)}\right\}^{*} u u_{\alpha}^{(1)}
$$

is a unitary operator in $\varepsilon_{p}^{-}(M)$.

Next we consider the case where $M^{G}$ is finite. We use the normalized dimension of projections. Assume $\operatorname{dim} e \geqq \operatorname{dim} e^{\prime}$. Take $n$ such that $n(\operatorname{dim} e) \geqq 1$ and take a projection $e_{0} \in M^{G}$ such that $e_{0} \leqq e$ and $\operatorname{dim} e_{0}=1 / n$. Take projections $e_{\alpha}^{(j)}$, $\alpha=1, \ldots, n, j=1,2$ such that $e_{\alpha}^{(j)} \in M^{G}, e_{\alpha}^{(j)} e_{\beta}^{(j)}=0$ for $\alpha \neq \beta, \operatorname{dim} e_{\alpha}^{(1)}=1 / n$ and $\operatorname{dim} e_{\alpha}^{(2)}$ $=\left(\operatorname{dim} e^{\prime}\right)\{n \operatorname{dim} e\}^{-1}$. Then there exist $u_{\alpha}^{(j)} \in M^{G}$ such that $\left\{u_{\alpha}^{(j)}\right\}^{*} u_{\alpha}^{(j)}=e_{\alpha}^{(j)}$, $u_{\alpha}^{(1)}\left\{u_{\alpha}^{(1)}\right\}^{*}=e_{0}$ and $u_{\alpha}^{(2)}\left\{u_{\alpha}^{(2)}\right\}^{*}=u e_{0} u^{*}$. Then the operator $U$ defined as before is an isometry in $\varepsilon_{p}^{-}(M)$. If $\operatorname{dim} e \leqq \operatorname{dim} e^{\prime}$, the same construction for $u^{*} \in \varepsilon_{q}^{-}(M)$ with $q=p^{-1}$ yields an isometry $U \in \varepsilon_{q}^{-}(M)$ and hence the co-isometry $U^{*}$ is in $\varepsilon_{p}^{-}(M)$.

Q.E.D.

We now present proof of Theorem 1(1). Since $g \in G$ commutes with $\varrho_{t}, \varepsilon_{p}$ also commutes with $\varrho_{t}$. Since $\varphi$ is $\varrho_{t}$-invariant, $\varphi^{-}$is also $\varrho_{t}$-invariant. Let $\varrho_{t}^{-}$be the continuous extension of $\varrho_{t}$ to $M, \sigma_{t}^{\varphi}$ be the modular automorphisms of $M$ [because we know that $\varphi^{-}$is faithful on $M$ due to Theorem 2(2)] relative to (the normal extension of) $\varphi^{-}$and

$$
\gamma_{t}=\varrho^{-}-t \cdot \sigma_{t}^{\bar{\phi}} \text {. }
$$

Since $\varphi^{-}$is $\varrho_{t}$-invariant, $\sigma_{t}^{\varphi^{-}}$commutes with $\varrho_{t}^{-}$. Hence $\gamma_{t}$ is a continuous oneparameter group of *automorphisms of $M$.

By definition $\varphi^{-}=\varphi \cdot \varepsilon_{1}$,

$$
\varphi^{-}\left(x \varrho_{t}(y)\right)=\varphi\left(\varepsilon_{1}(x) \varrho_{t}(y)\right)
$$


holds for $x \in \mathfrak{U}$ and $y \in \mathfrak{U}^{G}$. Since $\varphi$ is a $\varrho_{t}$-KMS state, $\varrho_{t}(y)$ satisfies the KMS condition for $\varphi^{-}$and hence

$$
\pi_{\bar{\varphi}}\left(\varrho_{t}(y)\right)=\sigma_{t}^{\varphi^{-}}\left(\pi_{\bar{\varphi}}(y)\right), y \in \mathfrak{A}^{G} .
$$

Hence $\gamma_{t}$ is an identity automorphism on $M^{G}$.

Let $\varepsilon_{p}^{-}(M) \neq 0$. Then $\varepsilon_{q}^{-}(M)=\varepsilon_{p}^{-}(M)^{*} \neq 0$ for $q=p^{-1}$. By Lemma 6 , either $\varepsilon_{p}^{-}(M)$ or $\varepsilon_{q}^{-}(M)$ has an isometry $U$. Suppose $U \in \varepsilon_{p}^{-}(M)$. Let $x \in \varepsilon_{p}^{-}(M)$. Then

$$
\gamma_{t}(x)=\gamma_{t}\left(x U^{*} U\right)=\gamma_{t}\left(x U^{*}\right) \gamma_{t}(U)=x U^{*} \gamma_{t}(U)=x u_{t}
$$

where $u_{t} \equiv U^{*} \gamma_{t}(U)$. (We have used $x U^{*} \in M^{G}$.)

Since $\varphi^{-}$is $G$-invariant, $\sigma_{t}^{\varphi^{-}}$commutes with $g^{-}$for all $g \in G$. Hence $\gamma_{t}$ commutes with $g^{-}$for all $g \in G$. Hence $\gamma_{t}(U) \in \varepsilon_{p}^{-}(M)$, which implies $u_{t} \in M^{G}$. Since $U U^{*} \in M^{G}$, we have $U U^{*}=\gamma_{t}\left(U U^{*}\right)$, which implies

$$
\begin{aligned}
& u_{t}^{*} u_{t}=\gamma_{t}\left(U^{*}\right) U U^{*} \gamma_{t}(U)=\gamma_{t}\left(U^{*}\right) \gamma_{t}\left(U U^{*}\right) \gamma_{t}(U)=1, \\
& u_{t} u_{t}^{*}=U^{*} \gamma_{t}(U) \gamma_{t}\left(U^{*}\right) U=U^{*} \gamma_{t}\left(U U^{*}\right) U=U^{*}\left(U U^{*}\right) U=1 .
\end{aligned}
$$

Hence $u_{t}$ is unitary. We also have

$$
u_{t} u_{s}=u_{t} \gamma_{t}\left(u_{s}\right)=U^{*} \gamma_{t}\left(U U^{*}\right) \gamma_{t+s}(U)=u_{t+s},
$$

due to $u_{s} \in M^{G}$. Hence $u_{t}$ is a continuous one-parameter group.

For $x \in M^{G}$, we have $U x \in \varepsilon_{p}^{-}(M)$ and hence

$$
U x u_{t}=\gamma_{t}(U x)=\gamma_{t}(U) x=U u_{t} x,
$$

where the last equality is due to $U \in \varepsilon^{-}(M)$, for example. By multiplying $U^{*}$ from the left, we obtain $x u_{t}=u_{t} x$ for all $x \in M^{G}$. Hence $u_{t}$ is in the center of $M^{G}$, which means that $u_{t}=\gamma_{t}(p) 1$. Therefore

$$
\gamma_{t}(x)=\gamma_{t}(p) x, x \in \varepsilon_{p}^{-}(M) \text {. }
$$

The Equation (5.7) defines $\gamma_{t}(p)$ for each $p \in H$. If $x \in \varepsilon^{-}(M)$ and $p \in H$, then $x^{*} \in \varepsilon_{q}^{-}(M)$ for $q=p^{-1}$ and $\gamma_{t}\left(p^{-1}\right)=\gamma_{t}(p)^{*}$. If $x \in \varepsilon_{p}^{-}(M)$ and $y \in \varepsilon_{q}^{-}(M)$ are both non-zero, then $x u y$ in the proof of Lemma 4 is a non-zero element of $\varepsilon_{p q}^{-}(M)$ and

$$
\gamma_{t}(p q) x u y=\gamma_{t}(x) \gamma_{t}(u) \gamma_{t}(y)=\gamma_{t}(p) \gamma_{t}(q) x u y
$$

and hence

$$
\gamma_{t}(p q)=\gamma_{t}(p) \gamma_{t}(q)
$$

Therefore $\gamma_{t} \in H^{\wedge}$. The dual $H^{\wedge}$ of $H$ can be identified with $G / G_{0}$ where $G_{0}$ is the subgroup of $G$ consisting of all $g \in G$ on which $p \in H$ takes the value 1. $\left(G_{0}=H^{\perp}\right.$.) By Lemma $5, H=G^{\wedge}$. Hence there exists a continuous one-parameter subgroup $\alpha_{t} \in G$ satisfying

$$
\gamma_{t} \pi_{\bar{\varphi}}=\pi_{\bar{\varphi}} \alpha_{t}
$$

on $\varepsilon_{p}(\mathfrak{A})$ for all $p$. Hence $\sigma_{t}^{\varphi^{-}} \pi_{\varphi}=\pi_{\varphi} \varrho_{t} \alpha_{t}$ and hence $\varphi^{-}$is $\varrho_{t} \circ \alpha_{t}$-KMS.

Q.E.D. 


\section{§6. Proof of Theorem 1 (2) and (3)}

We first prove that $\varphi$ satisfies Assumption B. For any $a \in \mathfrak{A}^{G}, p \in \Delta$ and $n$, there exists $F_{a, n} \in \mathscr{F}_{1}$ satisfying

$$
\begin{aligned}
& F_{a, n}(t)=\varphi^{\wedge}\left(a b_{n}^{(p)} \varrho_{t}\left(b_{n}^{(p)}\right)^{*}\right) p\left(\alpha_{t}\right)^{*}, \\
& F_{a, n}(t+i)=\varphi^{\wedge}\left(\varrho_{t}\left(b_{n}^{(p)}\right)^{*} a b_{n}^{(p)}\right) p\left(\alpha_{t}\right)^{*},
\end{aligned}
$$

by $\varrho_{t} \circ \alpha_{t}$-KMS condition for $\varphi \hat{\wedge}$. By Assumption A, there exists a subnet $n(v)$ such that limits in (4.3) exists. Then there exists $F_{x} \in \mathscr{F}_{1}$ satisfying

$$
\begin{aligned}
& F_{x}(t)=\left(\Omega, x f_{p}(t) \Omega\right) p\left(\alpha_{t}\right)^{*}, \\
& F_{x}(t+i)=\left(\Omega, x g_{p}(t) \Omega\right) p\left(\alpha_{t}\right)^{*},
\end{aligned}
$$

for $x=\pi_{\varphi}(a)$ and hence for any $x \in M^{G}$.

For positive elements $x \in M^{G} \cap M^{\prime}, F_{x}(t)$ and $F_{x}(t+i)$ are positive type functions of $t$ because (6.1) and (6.2) are positive type functions. Hence they vanish identically if they vanish at $t=0$. Since $\Omega$ is separating for $M$ by $\varrho_{t} \circ \alpha_{t}-\mathrm{KMS}$ condition for $\varphi^{\wedge}$, we have the equivalence of $x f_{p}(0)=0$ and $x g_{p}(0)=0$. Since $f_{p}(0)+g_{p}(0) \geqq 1$, these are equivalent to $x=0$. Hence the supports of $f_{p}(0)$ and $g_{p}(0)$ are both 1 .

Since $\Omega$ is separating for $M, M^{G}$ is isomorphic to its restriction to the closure of $M^{G} \Omega$. If a central projection $e$ of $M^{G}$ satisfies $e \pi_{\varphi^{(}}\left(\left(b_{n}^{(p)}\right)^{*} b_{n}^{(p)}\right)=0$ for all $n$, then $e f_{p}(0)=0$ and hence $e=0$. The same holds for $b_{n}^{(p)}\left(b_{n}^{(p)}\right)^{*}$. Therefore Assumption B holds for the restriction $\varphi$ of $\varphi^{\wedge}$ to $\mathfrak{A}^{G}$.

On $\mathfrak{U}^{G}$, the action of $\varrho_{t} \circ \alpha_{t}$ is the same as that of $\varrho_{t}$. Hence the statements of KMS condition relative to $\varrho_{t} \circ \alpha_{t}$ and relative to $\varrho_{t}$ coincide for elements in $\mathfrak{A}^{G}$. Therefore a restriction $\varphi$ of a $\varrho_{t} \circ \alpha_{t}-\mathrm{KMS}$ state $\varphi^{-}$of $\mathfrak{A}$ to $\mathfrak{U}^{G}$ is a $\varrho_{t}$-KMS state. If $\varphi^{\wedge}$ is primary, then $M^{G}$ is a factor by Theorem 2(1). Hence $\varphi$ is an extremal. $\varrho_{t}$-KMS state. If $\varphi^{\wedge}$ is $G$-invariant, then $\varphi^{\wedge}=\varphi^{\wedge} \circ g$ for all $g \in G$ and hence $\varphi^{\wedge}=$ $\varphi^{\wedge} \circ \varepsilon_{1}=\varphi \circ \varepsilon_{1}$. If $\varphi^{\wedge}$ is extremal among $G$-invariant $\varrho_{t} \circ \alpha_{t}-\mathrm{KMS}$ states, then $M^{G} \cap M^{\prime}$ is trivial by Lemma 1 . By Theorem $2(1), M^{G}$ is a factor and hence $\varphi$ is an extremal $\varrho_{t}$-KMS state. (3) follows from (1) and (2).

\section{§ 7. The Structure of the Cyclic Representation Associated with $\varphi$}

Let $\varphi$ be an extremal $\varrho_{t}$-KMS state of $\mathfrak{U}^{G}, \varphi^{-}$be its unique extention to a $G$ invariant state of $\mathfrak{A}, M=\pi_{\varphi}-(\mathfrak{U})^{\prime \prime}$ and $M^{G}=\pi_{\varphi}-\left(\mathfrak{A}^{G}\right)^{\prime \prime}$.

Theorem 3. Assume that $M^{G}$ is a factor. Let $H$ be the set of $p \in G^{\wedge}$ such that $\varepsilon_{p}^{-}\left(M \cap M^{\prime}\right) \neq 0$. Then $H$ is a subgroup.

Let $I$ be a subset of $G^{\wedge}$ containing 1 such that $I \cap g^{\wedge} H$ consists of one point for every $g^{\wedge} \in G^{\wedge}$. Let $E$ be the projection operator on the subspace $\sum_{q \in I} \mathfrak{H}_{q}$ where

$$
\mathfrak{H}_{q}=\text { the closure of } \varepsilon_{q}^{-}(M) \Omega,
$$

$\Omega$ being the cyclic vector associated with $\varphi^{-}$. Then there exists a unitary map $U$ from $\mathfrak{H}_{\varphi^{-}}$onto $L_{2}\left(H^{\wedge}\right) \otimes E \mathfrak{S}_{\varphi^{-}}$such that

$$
\begin{aligned}
U \Phi & =\sum_{p \in H} p_{\mathrm{vec}} \otimes E U_{p}^{*} \Phi, \\
U \Omega & =1_{\mathrm{vec}} \otimes \Omega, \\
U x U^{*} & =\sum_{p \in H} p_{\mathrm{op}} \otimes E U_{p}^{*} x E, x \in M,
\end{aligned}
$$


where $p_{\mathrm{vec}}$ is $p(h), h \in H^{\wedge}$, as a vector in $L_{2}\left(H^{\wedge}\right), p_{\mathrm{op}}$ is the same function as a multiplication operator and $U_{p}$ is a unitary representation of $H$ satisfying $U_{p} \in \varepsilon_{p}^{-}\left(M \cap M^{\prime}\right)$, which exists.

Proof. We divide the proof into several steps.

Step 1. The center $M \cap M^{\prime}$ is isomorphic to $L^{\infty}\left(H^{\wedge}\right)$ with $g^{-}$acting as the translation by $[g] \in G / G_{0} \sim H^{\wedge}$ and a unitary representation $U_{p}$ of $H$ corresponding to $p_{\text {op }}$ :

Since $M \cap M^{\prime}$ is $G$-invariant as a set, $\varepsilon_{p}^{-}\left(M \cap M^{\prime}\right)$ is in $M \cap M^{\prime}$ for all $p$. Since $M^{G}$ is a factor, any $u$ and $v$ in $\varepsilon_{p}^{-}\left(M \cap M^{\prime}\right)$ have the property that $u v^{*}$ and $v^{*} u$, being in the center of $M^{G}$, must be multiples of the identity. Hence each non-zero $\varepsilon_{p}^{-}\left(M \cap M^{\prime}\right)$ is a set of the form $\mathbb{C} u_{p}$ where $u_{p}$ is unitary and $\mathbb{C}$ denotes the complex numbers. Since 1 is in $\varepsilon^{-}\left(M \cap M^{\prime}\right)$, we take $u_{1}=U_{1}=1$. Since $u_{p_{1}} u_{p_{2}}^{*} \in \varepsilon_{q}^{-}\left(M \cap M^{\prime}\right)$ and $u_{p_{1}} u_{p_{2}}^{*} \neq 0$ for $q=p_{1} p_{2}^{-1}$, the set $H$ is a subgroup of $G^{\wedge}$.

Using the commutativity of $u_{p}, p \in H$, we can find complex numbers $\theta_{p}$ of modulus 1 such that $U_{p}=\theta_{p} u_{p}$ is a unitary representation of $H$ as follows. Suppose we have been able to choose $\theta_{p}$ for all $p$ in a subgroup $H_{1}$ of $H$. We shall prove that we can extend the choice $\theta_{p}$ (such that $U_{p}$ is a representation) for all $p$ in a subgroup $H_{2}$ generated by $H_{1}$ and an element $h$ of $H$. Since the obvious choice $\theta_{1}=1$ works for $H_{1}=\{1\}$, this will prove the existence of desired $\theta_{p}$ for all $p \in H$ by induction. To extend $\theta_{p}$ from $H_{1}$ to $H_{2}$, let $H_{10}$ be the intersection of $H_{1}$ and $h^{\mathbb{Z}}=\left\{h^{n} ; n=0, \pm 1, \ldots\right\}$. Obviously $H_{10}$ is a subgroup of $H_{1}$ and $h^{\mathbb{Z}}$ and is a set of the form $h^{m \bar{Z}}$. If $m=0$, we can choose $U_{p k}=U_{p}\left(u_{h}\right)^{n}$ for $p \in H_{1}$ and $k=h^{n}$. If $m>0$, we can choose $U_{p k}=U_{p} U_{h}^{n}$ for $p \in H_{1}$ and $k=h^{n}$ where $U_{h}$ is chosen so that $\left(U_{h}\right)^{m}=U_{\left(h^{m}\right)}, U_{\left(h^{m}\right)}$ being already defined due to $h^{m} \in H_{1}$. The consistency and representation property are immediate.

Since $M \cap M^{\prime}$ is generated by a faithful unitary representation of $H$, it is isomorphic to $L^{\infty}\left(H^{\wedge}\right)$ with $U_{p}$ corresponding to $p_{\mathrm{op}}$, where $H^{\wedge}$ can be identified with the compact group $G / G_{0}, G_{0}$ being closed as the annihilator of $H$. Since $g^{-}\left(U_{p}\right)=p(g) U_{p}, g^{-}$acts as the translation $p(k) \rightarrow p(k \cdot[g])$ where $[g]=g G_{0} \in G / G_{0}$.

Step 2. The convergence of the sum in (7.2) and unitarity of $U$.

For $x, y \in M$ and $p, q \in G^{\wedge}$, we have

$$
\begin{aligned}
\left(\varepsilon_{p}^{-}(x) \Omega, \varepsilon_{q}^{-}(y) \Omega\right) & =\int p(g) q\left(g^{\prime}\right)^{*}\left(x \Omega, U\left(g^{-1} g^{\prime}\right) y \Omega\right) d g d g^{\prime} \\
& =\int p\left(g_{1}\right)\left(p^{-1} q\right)\left(g^{\prime}\right)^{*}\left(x \Omega, U\left(g_{1}^{-1}\right) y \Omega\right) d g_{1} d g^{\prime}
\end{aligned}
$$

which vanishes for $p \neq q$. Hence the subspaces (7.1) are mutually orthogonal. Since $\varepsilon_{p}(\mathfrak{A}), p \in G^{\wedge}$, is total in $\mathfrak{A}$ and $\Omega$ is cyclic, we have

$$
\mathfrak{H}_{\varphi^{-}}=\sum_{q \in G^{\star}}{ }^{\oplus} \mathfrak{H}_{q}
$$

Let $\Phi=\sum_{q \in G^{\wedge}} \Phi_{q}$ with $\Phi_{q} \in \mathfrak{H}_{q}$. Each $q \in G^{\wedge}$ has a unique decomposition $q=p(q) r(q)$ with $p(q) \in H$ and $r(q) \in I$. Since $E U_{p}^{*} \Phi_{q}=0$ unless $p(q)=p$, the summation on the right hand side of (7.2) can be written as

$$
U \Phi=\sum_{p \in H} p_{\mathrm{vec}} \otimes\left(\sum_{r \in I} U_{p}^{*} \Phi_{p r}\right)
$$


Since $U_{p}^{*} \Phi_{p r} \in \mathfrak{H}_{r}, r \in I$, are mutually orthogonal for each fixed $p$, and since $p_{\text {vec }}$, $p \in H$, are mutually orthogonal, we obtain the absolute convergence of

$$
\|U \Phi\|^{2}=\sum_{p \in H} \sum_{r \in I}\left\|U_{p}^{*} \Phi_{p r}\right\|^{2}=\|\Phi\|^{2} .
$$

Hence the summation in (7.2) is strongly convergent for any $\Phi$. A similar calculation shows that $U$ is isometric:

$$
(U \Psi, U \Phi)=\sum_{p \in H} \sum_{r \in I}\left(U_{p}^{*} \Psi_{p r}, U_{p}^{*} \Phi_{p r}\right)=(\Psi, \Phi) .
$$

The vector $p_{\text {vec }}$ is a complete orthonormal basis in $L_{2}\left(H^{\wedge}\right)$. For each fixed $p$, any vector $\Phi_{0} \in E \mathfrak{G}_{\varphi^{-}}$can be written as $\Phi_{0}=\sum_{r \in I} \Phi_{0 r}, \Phi_{0 r} \in \mathfrak{H}_{r}$, and hence the choice $\Phi=\sum_{r \in I} U_{p} \Phi_{0 r}$ (with $\|\Phi\|^{2}=\sum_{r}\left\|\Phi_{0 r}\right\|^{2}=\left\|\Phi_{0}\right\|^{2}$ convergent) yields $U \Phi=p_{\text {vec }} \otimes \Phi_{0}$. Hence the image of $U$ contains a total set and hence $U$ is a unitary map onto $L^{\infty}\left(H^{\wedge}\right) \otimes E \mathfrak{H}_{\varphi^{-}}$.

Step 3. Proof of (7.3) and (7.4). The Equation (7.3) is a special case of (7.2). The Equation (7.4) follows from the following computation for $\Phi=\sum \Phi_{q}, \Phi_{q} \in \mathfrak{H}_{q}$ :

$$
\begin{aligned}
\left(U x U^{*}\right)(U \Phi) & =U x \Phi=\sum_{p \in H} p_{\mathrm{vec}} \otimes E U_{p}^{*} x \Phi \\
& =\sum_{p \in H} p_{\mathrm{vec}} \otimes E U_{p}^{*} x \sum_{p^{\prime} \in H} U_{p^{\prime}} \sum_{r \in I} U_{p^{\prime}}^{*} \Phi_{p^{\prime} r} \\
& =\sum_{p, p^{\prime}}\left(p p^{\prime-1}\right)_{\mathrm{op}} p_{\mathrm{vec}}^{\prime} \otimes E U_{p}^{*} U_{p^{\prime}} x \sum_{r \in I} U_{p^{\prime}}^{*} \Phi_{p^{\prime} r} \\
& =\sum_{p^{\prime \prime} p^{\prime}}\left(p_{\mathrm{op}}^{\prime \prime} \otimes E U_{p^{\prime \prime}}^{*} x E\right)\left(p_{\mathrm{vec}}^{\prime} \otimes \sum_{r \in I} U_{p^{\prime}}^{*} \Phi_{p^{\prime} r}\right) \\
& =\sum_{p^{\prime \prime}}\left(p_{\mathrm{op}}^{\prime \prime} \otimes E U_{p^{\prime \prime}}^{*} x E\right) U \Phi .
\end{aligned}
$$

Q.E.D.

Corollary. If $H=G^{\wedge}$, then $E \mathfrak{H}_{\varphi^{-}}=\mathfrak{H}_{1}$ and $U M U^{*}=L^{\infty}\left(H^{\wedge}\right) \otimes M_{E}^{G}$.

Proof. By definition of $E, E \mathfrak{H}_{\varphi^{-}}=\mathfrak{H}_{1}$. From (7.4). $U x U^{*}$ for $x \in M$ is clearly in $L^{\infty}\left(H^{\wedge}\right) \otimes E M E$. Since $M$ is generated by $\varepsilon_{q}^{-}(M), q \in G^{\wedge}$, and $E \varepsilon_{q}^{-}(M) E=0$ unless $q=1$, we have $E M E=E \varepsilon^{-}{ }_{1}(M) E=M^{G} E$.

We note that $M^{G} E$ is isomorphic to $M^{G}$ by the assumption that $M^{G}$ is a factor and $E \in\left(M^{G}\right)^{\prime}$.

\section{§ 8. Proof of Theorem 1(4)}

The existence of an extension of an extremal $\varrho_{t}$-KMS state $\varphi$ of $\mathfrak{A}^{G}$ to an extremal $\varrho_{t} \cdot \alpha_{t}-\mathrm{KMS}$ state $\varphi^{\wedge}$ of $\mathfrak{Q}$ is proved first in several steps:

Step 1. The case $H=G^{\wedge}$, where $H$ is defined for $\varphi^{-}$in Theorem 3.

Let $M$ and $M^{G}$ be as in Section 7. There exists a conditional expectation $\varepsilon$ from $M$ onto its center relative to the normal extension of $\varphi^{-}$: For $x \in M$, $\varepsilon(x) \in M \cap M^{\prime}$ is uniquely defined by

$$
(\Omega, \varepsilon(x) z \Omega)=(\Omega, x z \Omega), z \in M \cap M^{\prime} .
$$


For $a \otimes b$ in Corollary to Theorem 3, with $a \in L^{\infty}\left(H^{\wedge}\right)$ and $b \in M_{E}^{G}$, we have

$$
\varepsilon\left(U^{*}(a \otimes b) U\right)=U^{*}(a \otimes 1) U(\Omega, b \Omega) .
$$

In particular, for $x \in M^{G}$, we have $U x U^{*}=1_{\mathrm{op}} \otimes x E$ and hence

$$
\varepsilon(x)=(\Omega, x \Omega) 1 .
$$

Any element $g \in H^{\wedge} \sim G\left(H=G^{\wedge}\right.$ in the present case) defines a pure state $\chi_{g}$ on $C(G)$ which has an extension to a pure state $\chi_{g}^{-}$of $U\left(M \cap M^{\prime}\right) U^{*}\left(\chi_{g}\left(p_{\text {op }}\right)=p(g)\right)$. We define

$$
\varphi^{\wedge}(a)=\bar{\chi}^{-}\left(U \varepsilon\left\{\pi_{\varphi^{-}}(a)\right\} U^{*}\right), a \in \mathfrak{A} .
$$

From (8.4), $\varphi^{\wedge}$ is clearly a state of $\mathfrak{A}$. By (8.3),

$$
\varphi^{\wedge}(a)=\varphi(a), a \in \mathfrak{A}^{G} \text {. }
$$

Next we prove that $\varphi^{\wedge}$ is primary. Let $M_{0}$ be the *-subalgebra of $M$ generated by $\varepsilon_{q}^{-}(M), q \in G^{\wedge}$. [It is the linear hull of $\varepsilon_{q}^{-}(M), q \in G^{\wedge}$.] For $x \in M_{0}$, let

$$
\pi(x)=\sum_{p \in H} E U_{p}^{*} x E \in M_{E}^{G},
$$

where the sum converges because it reduces to a single term $p=q$ for $x \in \varepsilon_{q}^{-}(M)$. For $x_{i} \in \varepsilon_{q_{i}}^{-}(M), i=1,2$, we have

$$
\begin{aligned}
\pi\left(x_{1}\right) \pi\left(x_{2}\right) & =U_{q_{1}}^{*} x_{1} U_{q_{2}}^{*} x_{2} E \\
& =U_{q_{1} q_{2}}^{*} x_{1} x_{2} E=\pi\left(x_{1} x_{2}\right), \\
\pi\left(x_{1}\right)^{*} & =x_{1}^{*} U_{q_{1}} E=U_{q_{1}} x_{1}^{*} E=\pi\left(x_{1}^{*}\right),
\end{aligned}
$$

and hence $\pi$ is a ${ }^{*}$-representation. Obviously

$$
\begin{aligned}
(\Omega, \pi(x) \Omega) & =\sum_{p \in H}\left(\Omega, E U_{p}^{*} x E \Omega\right) \\
& =\chi^{-}\left(U \varepsilon(x) U^{*}\right) .
\end{aligned}
$$

Since $\pi\left(M^{G}\right) \Omega=M_{E}^{G} \Omega$ is dense in $E \mathfrak{G}=\mathfrak{H}_{1}, \pi \circ \pi_{\varphi^{-}}$and $\Omega$ are the cyclic representation and the cyclic vector associated with the restriction of $\varphi^{\wedge}$ defined by (8.4) to the subalgebra $\mathfrak{U}_{0}$ of $\mathfrak{A}$ generated by $\varepsilon_{q}(\mathfrak{Q}), q \in G^{\wedge}$. Since $\varphi^{\wedge}$ is a state of $\mathfrak{U}$ and $\mathfrak{A}_{0}$ is dense in $\mathfrak{A}, \pi \circ \pi_{\varphi^{-}}$has a continuous extension to the cyclic representation of $\mathfrak{A}$ associated with $\varphi^{\wedge}$. We already know by (8.6) that $\pi\left\{\pi_{\varphi^{-}}\left(\mathfrak{I}_{0}\right)\right\}$ is in $M_{E}^{G}$. Since $\pi\left(\pi_{\varphi^{-}}\left(\mathfrak{H}^{G}\right)\right)=\pi_{\varphi^{-}}\left(\mathfrak{Q}^{G}\right)_{E}$, we have $\pi\left(\pi_{\varphi^{-}}\left(\mathfrak{Q}^{G}\right)\right)^{\prime \prime}=M_{E}^{G}$, which is a factor. Hence $\varphi^{\wedge}$ is primary.

Finally, we prove that $\varphi^{\wedge}$ is a $\varrho_{t} \circ \alpha_{t}-\mathrm{KMS}$ state. Since $\varphi^{-}$is a $\varrho_{t} \circ \alpha_{t}-\mathrm{KMS}$ state of $\mathfrak{A}$, the modular automorphisms of $L^{\infty}\left(H^{\wedge}\right) \otimes M_{E}^{G}$ relative to $1_{\text {vec }} \otimes \Omega$ induces the automorphisms $U \pi_{\varphi^{-}}(a) U^{*} \rightarrow U \pi_{\varphi^{-}}\left(\varrho_{t} \circ \alpha_{t}(a)\right) U^{*}$. On the other hand, the modular operator for $1_{\text {vec }} \otimes \Omega$ is $1 \otimes \Delta_{\Omega}$ where $\Delta_{\Omega}$ is the modular operator for $\Omega$ and $M^{G}$, because $L^{\infty}\left(H^{\wedge}\right)$ is commutative. Since $\varphi$ is a $\varrho_{t}$ - KMS state, $\Delta_{\Omega}^{i t} x \Delta_{\Omega}^{-i t}=\varrho_{t}^{-}(x)=$ $\varrho_{t^{-}}^{-} \alpha_{t}^{-}(x)$ for $x \in M^{G}$. Since $\Delta_{\Omega}^{i t}$ induces the modular automorphisms of $\varphi^{\wedge}$, we have

$$
\begin{aligned}
\sigma_{t}^{\varphi^{\dagger}}\{\pi(x)\} & =\Delta_{\Omega}^{i t} U_{p}^{*} x E \Delta_{\Omega}^{-i t} \\
& =\varrho^{-}\left(U_{p}^{*} x\right) E=\varrho^{-}{ }_{t}^{-} \alpha_{t}^{-}\left(U_{p}^{*}\right) \varrho_{t}{ }_{t}^{-} \alpha_{t}^{-}(x) E
\end{aligned}
$$


for $x \in \varepsilon_{p}^{-}(M)$. Since $U_{p}^{*}$ is in the center and $\varphi^{-}$is a $\varrho_{t} \circ \alpha_{t}-\mathrm{KMS}$ state, $\varrho_{t}^{-} \alpha_{t}^{-}\left(U_{p}^{*}\right)=U_{p}^{*}$. Hence

$$
\sigma_{t}^{\varphi^{-}}(\pi(x))=U_{p}^{*} \varrho_{t}^{-} \alpha_{t}^{-}(x) E=\pi\left(\varrho_{t}^{-} \alpha_{t}^{-}(x)\right) .
$$

This shows that $\varphi^{\wedge}$ is a $\varrho_{t} \circ \alpha_{t}$-KMS state. Since it is primary, we have shown the existence of an extremal $\varrho_{t} \circ \alpha_{t}-\mathrm{KMS}$ state $\varphi^{\wedge}$, whose restriction to $\mathfrak{A}^{G}$ is the given $\varphi$, for the case $H=G^{\wedge}$.

Step 2. The general case.

Let $G_{0}=H^{\perp}$ [the set of $g \in G$ such that $p(g)=1$ for all $\left.p \in H\right]$. Let $\mathfrak{Q}^{G_{0}}$ be the set of $a \in \mathfrak{U}$ satisfying $g(a)=a$ for all $g \in G_{0}$. It is $G$-invariant as a set. The $G$-invariant extension of $\varphi$ to $\mathfrak{A}^{G_{0}}$ is the restriction of $\varphi^{-}$to $\mathfrak{H}^{G_{0}}$ and the associated cyclic representation is obtained by restricting $\pi_{\varphi^{-}}(a), a \in \mathfrak{H}^{G_{0}}$, to the subspace $E_{0} \mathfrak{H}=$ $\sum_{p \in H} \mathfrak{H}_{p^{*}}$. Obviously $\mathfrak{A}^{G_{0}}$ is generated by $\varepsilon_{p}(\mathfrak{A}), p \in H$, and hence $\pi_{\varphi^{-}}\left(\mathfrak{H}^{G_{0}}\right)^{\prime \prime}$ is generated by $U_{p}, p \in H$ and $\mathfrak{H}^{G}$. Hence by Step 1 , there exists an extremal $\varrho_{t} \circ \alpha_{t}$-KMS state $\hat{\varphi}^{\wedge}{ }_{0}$ of $\mathfrak{Q}^{G_{0}}$ whose restriction to $\mathfrak{H}^{G}$ is $\varphi$.

We now show that the unique $G_{0}$-invariant extension

$$
\varphi^{\wedge}(a)=\varphi_{0}\left(\int_{G_{0}} g a d g\right), \quad a \in \mathfrak{A},
$$

is an extremal $\varrho_{t} \circ \alpha_{t}-\mathrm{KMS}$ state of $\mathfrak{A}$, whose restriction to $\mathfrak{A}^{G}$ is $\varphi$.

Since $\varrho_{t} \circ \alpha_{t}$ commutes with all $g \in G, \varepsilon_{q_{i}}(\mathfrak{R})$ is $\varrho_{t} \circ \alpha_{t}$-invariant as a set. Let $a_{i} \in \varepsilon_{q_{i}}(\mathfrak{2}), \varrho_{t} \circ \alpha_{t}\left(a_{2}\right)=a_{2}(t)$. We have

$$
\begin{aligned}
\varphi^{\wedge}\left(a_{1} a_{2}(t)\right) & =0 \quad \text { if } \quad q_{1} q_{2} \notin H \\
& =\varphi^{\hat{\prime}}\left(a_{1} a_{2}(t)\right) \text { if } q_{1} q_{2}=p \in H .
\end{aligned}
$$

The $G$-invariant extension $\varphi_{0}^{-}$of $\varphi$ to $\mathfrak{A}^{G_{0}}$ is obviously the restriction (to $\mathfrak{A}^{G_{0}}$ ) of the $G$-invariant extension $\varphi^{-}$(to $\left.\mathfrak{A}\right)$ of $\varphi$. Hence the restriction of $\pi_{\varphi^{-}}$to the cyclic subspace $\left\{\pi_{\varphi^{-}}\left(\mathfrak{H}^{G_{0}}\right) \Omega\right\}^{-}$is the cyclic representation associated with $\varphi^{-}{ }_{0}$. Hence by (8.7) we obtain for $q_{1} q_{2} \equiv p \in H$

$$
\begin{aligned}
\varphi_{0}\left(a_{1} a_{2}(t)\right) & =\varphi^{-}\left(U_{p}^{*} \pi_{\varphi^{-}}\left(a_{1} a_{2}(t)\right)\right) \\
& =\left(\Omega, U_{p}^{*} \pi_{\varphi^{-}}\left(a_{1}\right) \sigma_{t}^{\varphi^{-}}\left(\pi_{\varphi^{-}}\left(a_{2}\right)\right) \Omega\right) .
\end{aligned}
$$

Similarly

$$
\begin{aligned}
\varphi^{\uparrow}\left(a_{2}(t) a_{1}\right) & =\left(\Omega, U_{p}^{*} \sigma_{t}^{\varphi^{-}}\left(\pi_{\varphi^{-}}\left(a_{2}\right)\right) \pi_{\varphi^{-}}\left(a_{1}\right) \Omega\right) \\
& =\left(\Omega, \sigma_{t}^{\varphi^{-}}\left(\pi_{\varphi^{-}}\left(a_{2}\right)\right) U_{p}^{*} \pi_{\varphi^{-}}\left(a_{1}\right) \Omega\right) .
\end{aligned}
$$

Hence $\varphi^{\wedge}\left(a_{1} \varrho_{t} \circ \alpha_{t}\left(a_{2}\right)\right)$ satisfies the statement of the KMS-condition for all $a_{1}$ and $a_{2}$ in a dense *-subalgebra $\mathfrak{A}_{0}$ of $\mathfrak{Q}$. By a standard argument, $\varphi^{\wedge}$ satisfies the KMS-condition relative to $\varrho_{t} \circ \alpha_{t}$.

We prove that $\varphi^{\wedge}$ is extremal by showing that it is primary in the next step.

Step $3 . \varphi^{\wedge}$ is primary.

We use the notation of Theorem 3 and its proof. For $x \in M_{0}$, we define

$$
\pi(x)=\sum_{p \in H} E U_{p}^{*} x E .
$$


For $x \in \varepsilon_{q}^{-}(M)$, the convergence of the sum is seen by the following computation:

$$
\left\|\sum_{p \in H} E U_{p}^{*} x E \Phi\right\|^{2}=\sum_{r \in I}\left\|U_{p(q, r)}^{*} x \Phi_{r}\right\|^{2} \leqq\|x\|^{2}\|\Phi\|^{2}
$$

where $E \Phi=\sum \Phi_{r}, \Phi_{r} \in \mathfrak{H}_{r}$ and $p(q, r) \in H$ is uniquely determined by $p(q, r)^{-1} q r \in I$. Since $U_{p}$ is in the center of $M$ and $U_{p^{-1}}=U_{p}^{*}, \pi(x)^{*}=\pi\left(x^{*}\right)$ follows. We also have

$$
\begin{aligned}
\pi\left(x_{1}\right) \pi\left(x_{2}\right) & =\sum_{p_{1}, p_{2}} E U_{p_{1}}^{*} x_{1} E U_{p_{2}}^{*} x_{2} E \\
& =\sum_{p, p_{2}} E U_{p}^{*} x_{1} U_{p_{2}} E U_{p_{2}}^{*} x_{2} E \\
& =\sum_{p} E U_{p}^{*} x_{1} x_{2} E=\pi\left(x_{1} x_{2}\right)
\end{aligned}
$$

for $x_{1}, x_{2} \in M$ where $U_{p_{2}} E U_{p_{2}}^{*}$ is the projection operator onto $\sum_{r \in I} \mathfrak{H}_{r p_{2}}$ and its sum over $p_{2} \in H$ is 1 . Hence $\pi$ is a ${ }^{*}$-representation of $M_{0}$.

Since $\pi\left(\varepsilon_{r}^{-}(M)\right) \Omega=\varepsilon_{r}^{-}(M) \Omega, r \in I, \Omega$ is cyclic for $\pi\left(M_{0}\right)$ in $E \mathfrak{S}$ and hence cyclic for $\pi \circ \pi_{\varphi}\left(\mathfrak{U}_{0}\right)$ in $E \mathfrak{H}$. Since

$$
(\Omega, \pi(x) \Omega)=\sum_{p \in H}\left(\Omega, U_{p}^{*} x \Omega\right)
$$

which vanishes for $x \in \mathcal{\varepsilon}_{q}^{-}(M), q \notin H$ and coincides with $\varphi_{{ }_{0}}(a)$ for $x=\pi_{\varphi^{-}}(a)$, $a \in \varepsilon_{p}(\mathfrak{A}), p \in H$ [or $\left.a \in\left(\mathfrak{U}^{G_{0}}\right)_{0}\right], \pi \circ \pi_{\bar{\varphi}}$ has a continuous extension to a cyclic representation of $\mathfrak{A}$ with the cyclic vector $\Omega$ associated with $\varphi^{\wedge}$.

For $x \in \varepsilon_{q}^{-}(M)$, we have $\pi(x)^{*} \pi(x)=\pi\left(x^{*} x\right)=x^{*} x E$. Since $M^{G}$ is isomorphic to $M_{E}^{G}$ and $x^{*} x \in M^{G}, \pi$ is faithful on each $\varepsilon_{q}^{-}(M)$ and $\|\pi(x)\|=\|x\|$. Let $x_{\alpha}$ be a uniformly bounded net in $\varepsilon_{q}^{-}(M)$ such that $\pi\left(x_{\alpha}\right)$ is weakly convergent. Then $\pi\left(x_{\alpha}\right) \Omega=U_{p}^{*} x_{\alpha} \Omega$ is weakly convergent for a fixed $p$ satisfying $p^{-1} q \in I$. Hence $x_{\alpha} y \Omega$ is weakly convergent for all $y \in M^{\prime}$. Since $\Omega$ is separating for $M$ due to Theorem 2(2), $M^{\prime} \Omega$ is dense and hence the uniformly bounded net $x_{\alpha}$ is convergent. Hence $w-\lim x_{\alpha}=$ $x \in \varepsilon_{q}^{-}(M)$ and $\pi\left(x_{\alpha}\right)$ is weakly convergent to $\pi(x)$. This shows that the unit ball of $\pi\left(\varepsilon_{q}^{-}(M)\right)$ is weakly closed. Since $\pi\left(\varepsilon_{q}^{-}(M)\right)$ is linear, it is weakly closed.

Let

$$
\begin{aligned}
\varepsilon_{s}^{0}(a) & =\int_{G_{0}} g(a) s(g)^{*} d g, \\
\varepsilon_{s}^{-0}(x) & =\int_{G_{0}} g^{-}(x) s(g)^{*} d g,
\end{aligned}
$$

where $s \in G_{0}^{\wedge}, a \in \mathfrak{A}, x \in M$. In particular $\varepsilon_{1}^{0}(\mathfrak{U})=\mathfrak{U}^{G_{0}}$ and $\varepsilon_{1}^{-0}(M)=\pi_{\bar{\varphi}}\left(\mathfrak{A}^{G_{0}}\right)^{\prime \prime} \equiv M^{G_{0}}$. The dual $G^{\wedge}$ of $G_{0}=H^{\perp}$ can be identified with $G^{\wedge} / H$, the restriction of $q \in G^{\wedge}$ to $G_{0}$ being the corresponding element of $G^{\wedge}$. Each $\varepsilon_{s}^{0}(\mathfrak{A})$ and $\varepsilon_{s}^{-0}(M)$ are generated by $\varepsilon_{q}(\mathfrak{A}), q H=s$, and $\varepsilon_{q}^{-}(M), q H=s$, respectively. Since $\pi\left(\varepsilon_{q}^{-}(M)\right)=\pi\left(\varepsilon_{q p}^{-}(M)\right), p \in H$ [because $\varepsilon_{q p}^{-}(M)=U_{p} \varepsilon_{q}^{-}(M)$ ], we see that the weak closure of $\pi \circ \pi_{\varphi^{-}}\left(\varepsilon_{s}^{0}(\mathfrak{A})\right)$ is $\pi\left(\varepsilon_{q}^{-}(M)\right)$ for any one $q$ satisfying $q(g)=s(g)$ for $g \in G_{0}$.

For $q \notin H, \varepsilon_{q}^{-}(M)$ does not contain non-zero central element. Hence for any $x \in \varepsilon_{q}^{-}(M)$, there exists $q^{\prime} \in G^{\wedge}$ and $y \in \varepsilon_{q}^{-}(M)$ such that $[x, y]$ is a non-zero element of $\varepsilon_{q q^{\prime}}(M)$. Hence $[\pi(x), \pi(y)]=\pi([x, y])$ is a non-zero element of $\pi\left(\varepsilon_{q q^{\prime}}^{-}(M)\right)$. Hence the weak closure of $\pi \circ \pi_{\varphi^{-}}\left(\varepsilon_{s}^{0}(\mathfrak{U})\right)$ for $s \neq 1$ does not contain any non-zero 
central element. We already know that $\pi \circ \pi_{\varphi^{-}}\left(\mathfrak{A}^{G_{0}}\right)^{\prime \prime}$ is a factor [and is equal to $\left.\pi\left(M^{G}\right)\right]$. By the analysis of the center of $M$ in the proof of Theorem 3, applied to the $G_{0}$-invariant extension $\varphi^{\wedge}$ of an extremal $\varrho_{t}{ }^{\circ} \alpha_{t}-\mathrm{KMS}$ state $\varphi^{\wedge}{ }_{0}$ of $\mathfrak{A}^{G_{0}}$ to $\mathfrak{A}$, shows then that the cyclic representation $\pi \circ \pi_{\varphi^{-}}$associated with $\varphi^{\wedge}$ is primary, i.e. its weak closure is a factor.

Q.E.D.

The rest of Theorem 1(4) is proved as follows: Since the restriction of $\varphi^{\wedge}$ to $\mathfrak{A}^{G}$ is $\varphi$, it is immediate that $\varphi^{-}=\int \varphi^{\wedge} \circ g d g$. Let $\varepsilon$ be the conditional expectation from $M$ onto its center relative to $\Omega$. Since (7.4) implies $U U_{p} U^{*}=p_{\text {op }} \otimes 1$, the center of $M$ is mapped onto $L^{\infty}\left(H^{\wedge}\right) \otimes 1$ by the unitary map $U$. From (7.4), it is then immediately seen that for $x \in M_{0}$

$$
\varepsilon(x)=U^{*}\left\{\sum_{p \in H}\left(\Omega, U_{p}^{*} x \Omega\right)\left(p_{\mathrm{op}} \otimes 1\right)\right\} U .
$$

By comparing (8.17) with (8.14), we see that

$$
\bar{\chi}_{1}^{-}\left\{U \varepsilon\left(\pi_{\varphi^{-}}(a)\right) U^{*}\right\}=\varphi^{\wedge}(a) \text {, }
$$

which shows that $\varphi^{-}=\int \varphi^{\wedge} \circ g d g$ is the central decomposition of a state in usual sense.

Both $\varphi^{\wedge} \circ g_{1}$ and $\varphi^{\wedge} \circ g_{2}$ are extremal $\varrho_{t} \circ \alpha_{t}$-KMS states. It is known that two extremal KMS states are disjoint unless they are the same. Since $\varphi^{\wedge}$ is not identically zero on $\varepsilon_{p}(\mathfrak{Q}), p \in H, \varphi^{\wedge} \circ g_{1}$ and $\varphi^{\wedge} \circ g_{2}$ are different unless $p\left(g_{1}\right)=p\left(g_{2}\right)$ for all $p \in H$, i.e. unless $g_{1} g_{2}^{-1} \in G_{0}$. On the other hand $\varphi^{\wedge}$ is $G_{0}$-invariant. Hence $\varphi^{\wedge} \circ g_{1}$ and $\varphi^{\wedge} \circ g_{2}$ are equal if $g_{1} g_{2}^{-1} \in G_{0}$ and are disjoint otherwise.

\section{§ 9. Additional Remarks}

1. Sectors. By Lemma 6, there exists either isometric or co-isometric operator in each $\varepsilon_{q}^{-}(M)$. If $M^{G}$ is properly infinite, then there exists a unitary operator in each $\varepsilon_{q}^{-}(M)$. If $M^{G}$ is finite, then this is not the case in general, as is known for homogeneous states of type $\mathrm{III}_{\lambda}$ factors $[3,1]$. For a non-zero partial isometry $u$ in $\varepsilon_{q}^{-}(M)$, the ratio

$$
d_{q} \equiv \operatorname{dim} u u^{*} / \operatorname{dim} u^{*} u>0
$$

is independent of $u$ and depends only on $q$. From $u_{1} v u_{2} \in \varepsilon_{q_{1} q_{2}}^{-}(M)$ for $u_{i} \in \varepsilon_{q_{i}}^{-}(M)$ and $v \in M^{G}$, it easily follows that $d_{q_{1}} d_{q_{2}}=d_{q_{1} q_{2}}$. Hence $q \in G^{\wedge} \rightarrow d_{q}>0$ is a group homomorphism. A special situation arises if $G$ is a one-dimensional torus. Then $G^{\wedge}$ is the group of integers. Hence either $d_{q}=1$ for all $q$ or $d_{q} \neq 1$ for all $q \neq 1$. The condition $d_{q}=1$ is obviously necessary and sufficient for $\varepsilon_{q}^{-}(M)$ to contain a unitary operator.

Since $\pi_{\varphi^{-}}\left(\mathfrak{O}^{G}\right)^{\prime \prime}=M^{G}$ is a factor, representations of $\mathfrak{Q}^{G}$ in different sectors $\mathfrak{S}_{q}, q \in G^{\wedge}$, are all quasi-equivalent. If there exists a unitary $u_{q}$ in $\varepsilon_{q}^{-}(M), q \in G^{\wedge}$, then representations of $\mathfrak{A}^{G}$ in sectors $\mathfrak{H}_{q_{1}}$ and $\mathfrak{S}_{q_{2}}$ are unitarily equivalent through $j_{\Omega}\left(u_{q}\right) \in M^{\prime}$ if $q_{2}=q q_{1}$ [because the modular conjugation operator $J_{\Omega}$ and $U(g)$ defined in Section 3 commute]. Thus representations of $\mathfrak{A}^{G}$ in all sectors are unitarily equivalent if $M^{G}$ is properly infinite, while representations of $\mathfrak{A}^{G}$ in sectors with the same $d(q)$ are unitarily equivalent if $M^{G}$ is finite. In the case of finite $M^{G}$, the restriction of $M^{G}$ to $\mathfrak{H}_{1}$ has a cyclic and separating vector $\Omega$ and 
hence has the coupling number 1 . If $d(q)>1$, then there exists a co-isometry $u$ in $\varepsilon_{q}^{-}(M)$ and $\operatorname{dim} u^{*} u=d(q)^{-1}$. By the transformation $u^{*}, \mathfrak{H}_{q}$ is mapped to $u^{*} u \mathfrak{S}_{1}$, the restriction of $M^{G}$ to $\mathfrak{S}_{q}$ and its commutant are mapped to the restriction of $M^{G}$ to $u^{*} u \mathfrak{H}_{1}$ and its commutant and hence $M^{G}$ on $\mathfrak{H}_{q}$ has the coupling number $d(q)$. This implies that representations of $\mathfrak{A}^{G}$ in sectors with different $d(q)$ are not unitarily equivalent.

\section{The case where Assumption B is violated. We first give typical examples:}

Example 1. Let $\mathfrak{A}$ be the CAR algebra for a non-relativistic Fermion. Let $\varrho_{t}$ be trivial. Let $G$ be the group $T=\mathbb{R} \bmod 2 \pi$ of gauge transformations $\alpha_{\theta}(\psi)=e^{i \theta} \psi$. Assumption $A$ is satisfied by asymptotic abelian property under spatial translations, $\Delta$ consisting of one character $p_{1}\left(\alpha_{\theta}\right)=e^{i \theta}$ and $b_{n}^{(p)}$ for $p=p_{1}$ being $b_{n}=\psi\left(f_{n}\right)$ with support of $f_{n}$ tending to infinity. Consider the Fock vacuum state $\omega$. The restriction $\omega_{0}$ of $\omega$ to $\mathfrak{Q}^{G}$ is a character of $\mathfrak{Q}^{G}$ yielding one-dimensional representation of $\mathfrak{Q}^{G}$. Hence $\omega_{0}$ is tracial and is an extremal $\varrho_{t}$-KMS state (for any temperature) of $\mathfrak{A}^{G}$. However Assumption B is violated because $\omega_{0}$ vanishes on $b_{n}^{*} b_{n}$ for all $n$. $\omega$ is not faithful on $\mathfrak{A}$ and has a one sided spectrum as far as the associated representation of $G=T$ is concerned.

Example 2. Let $\mathscr{B}$ be a $C^{*}$-algebra with a continuous one-parameter group $\eta_{t}$ of *-automorphisms and an extremal $\eta_{t}$-KMS state $\omega_{1}$. Consider the algebra $\mathfrak{A} \otimes \mathscr{B}$ with $\varrho_{t}=\imath \otimes \eta_{t}$ and $G=T \otimes \imath$. Assumption A is satisfied with $b_{n} \otimes 1$ playing the role of $b_{n}^{(p)}$ for $p=p_{1}$. The state $\varphi=\omega_{0} \otimes \omega_{1}$ is an extremal $\varrho_{t}$-KMS state of $(\mathfrak{H} \otimes \mathscr{B})^{G}$ $=\mathfrak{U}^{T} \otimes \mathscr{B}$.

At least some aspect of these examples is quite general. Consider the case where $G=T$, Assumption A is satisfied by some $b_{n}=b_{n}^{(p)}$ for $p=p_{1}$ and $\varphi$ is an extremal $\varrho_{t}$-KMS state of $\mathfrak{A}^{G}$. Let $\mathfrak{H}_{q}$ be defined by (7.1) for the $G$-invariant exten$\operatorname{sion} \varphi^{-}$of $\varphi$ to $\mathscr{H}$. Let $E_{q}$ be the orthogonal projection on $\mathfrak{H}_{q}, q \in G^{\wedge}$. The set of $q \in G^{\wedge}$ satisfying $\mathfrak{H}_{q} \neq 0$ is called the spectrum of (the representation of) $G$ on $\mathfrak{G}_{\varphi^{-}}$.

If Assumption B is satisfied, then this spectrum is $G^{\wedge}$ consisting of $p_{n}\left(\alpha_{\theta}\right)=e^{i n \theta}$, $n \in \mathbb{Z}$. We now prove the following assertion:

If Assumption B is violated, then the spectrum of $G$ on $\mathfrak{H}_{\varphi^{-}}$consists of $p_{n}$ with $n \geqq 0$ alone or $p_{n}$ with $n \leqq 0$ alone. Furthermore $E_{q} \in M$ and $M$ is a factor.

Proof of this assertion is as follows: From Assumption A, $f_{p}(t)$ and $g_{p}(t)$ of (4.3) are in $M^{G} \cap M^{\prime}$, which must be multiples of the identity operator by Lemma 2. Since $\varphi$ is primary, Assumption B is violated only if $\varphi\left(b_{n}^{*} b_{n}\right)=0$ for all $n$ or $\varphi\left(b_{n} b_{n}^{*}\right)=0$ for all $n$. Hence $g_{p_{1}}(t) \equiv 0$ or $f_{p_{1}}(t) \equiv 0$. By Assumption A, $f_{p_{1}}(0) \neq 0$ or $g_{p_{1}}(0) \neq 0$.

Consider the case $g_{p_{1}}(t)=0, f_{p_{1}}(0) \neq 0$. By the same argument as proof of Lemma 3, we obtain $\varphi\left(a^{*} a\right)=0$ for any $a \in \varepsilon_{p}(\mathfrak{R})$ with $p=p_{n}, n>0$. Hence the spectrum of $G$ on $\mathfrak{H}_{\varphi^{-}}$is one-sided, consisting of $p_{n}$ with $n \leqq 0$ alone.

If $y \in M^{\prime}$ and $x \in M$, we have

$\left(\varepsilon_{p}^{-}(x) \Omega, y \Omega\right)=\left(\Omega, y \varepsilon_{p}^{-}(x)^{*} \Omega\right)=0$

for $p=p_{n}, n<0$ because $\overline{\varepsilon_{p}}(x)^{*} \Omega \in \mathfrak{H}_{p_{-n}}=0$. Hence $y \Omega \in \mathfrak{H}_{p_{0}}$ for all $y \in M^{\prime}$. This implies

$$
U(g) y U(g)^{*} \Omega=U(g) y \Omega=y \Omega, y \in M^{\prime}
$$


where $U(g)$ is defined by (3.2). Since $U(g) y U(g)^{*} \in M^{\prime}$ for $y \in M^{\prime}$ and since $\Omega$ is separating for $M^{\prime}$ (being cyclic for $M$ ), we have $[U(g), y]=0$. Hence $U(g) \in M$ and $E_{q} \in M$.

Since $\varphi$ is an extremal KMS state, the restriction of $M$ to $\mathfrak{S}_{p_{0}}$ is a factor. Hence the restriction of $M^{\prime}$ to $\mathfrak{S}_{p_{0}}$ is also a factor, due to $\left(M^{\prime}\right)_{E_{p_{0}}}=\left(M_{E_{p_{0}}}\right)^{\prime}$. Since $\Omega$ is separating for $M^{\prime}$, the restriction of $M^{\prime}$ to $\mathfrak{H}_{p_{0}}$ is isomorphic to $M^{\prime}$. Hence $M^{\prime}$ is a factor, which implies that $M$ is a factor.

The other alternative $f_{p_{1}}(0)=0$ can be discussed similarly.

\section{Uniqueness up to gauge for extension to clustering state.}

If a state is invariant and ergodic (i.e. an extremal invariant state) under a group of automorphisms such as $\tau_{t}, t \in R$, or $\tau_{n}, n \in \mathbb{Z}$, then it satisfies a weak clustering property:

$$
\eta\left(\psi\left(x \tau_{t}(y)\right)-\psi(x) \psi(y)\right\}=0,
$$

where $\eta\left(A_{t}\right) \equiv \lim T^{-1} \int_{0}^{T} A_{t}$ or $\lim N^{-1} \sum_{n=1}^{N} A_{n t_{0}}$.

If a sequence of *-automorphisms $\tau_{n}$ of $\mathfrak{A}$ satisfies

$$
\lim \left\|\left[x, \tau_{n}(y)\right]\right\|=0, x, y \in \mathfrak{A},
$$

then any primary state $\psi$ of $\mathfrak{A}$ satisfies

$$
\lim _{n}\left\{\psi\left(x \tau_{n}(y)\right)-\psi(x) \psi(y)\right\}=0 .
$$

Let us call $\psi$ satisfying (9.2) or (9.4) clustering.

If every $g \in G$ commutes with $\varrho_{t}$ or $\tau_{n}$ above, then the following holds:

Proposition (Haag-Kastler). If clustering states $\psi_{1}$ and $\psi_{2}$ of $\mathfrak{A}$ have the same restriction to $\mathfrak{Q}^{G}$, then $\psi_{2}=\psi_{1} \circ \mathrm{g}$ for some $g \in G$.

Proof. Let $H_{i}$ be the subset of $G^{\wedge}$ consisting of all $p$ satisfying $\psi_{i}(x) \neq 0$ for some $x \in \varepsilon_{p}(\mathfrak{H})$. Since $x^{*} \tau_{n}(x) \in \mathfrak{A}^{G}$,

$$
\left|\psi_{1}(x)\right|^{2}=\eta\left\{\psi_{1}\left(x^{*} \tau_{n}(x)\right)\right\}=\eta\left\{\psi_{2}\left(x^{*} \tau_{n}(x)\right)\right\}=\left|\psi_{2}(x)\right|^{2} .
$$

Hence $H_{1}=H_{2} \equiv H$.

If $\psi_{1}\left(e_{p}\right) \neq 0$ for an $e_{p} \in \varepsilon_{p}(\mathfrak{g})$, we define

$$
c_{p}=\psi_{2}\left(e_{p}\right) / \psi_{1}\left(e_{p}\right) \text {. }
$$

By (9.5), $\left|c_{p}\right|=1$.

Let $x \in \varepsilon_{p}(\mathfrak{Q})$. Then

$$
\psi_{1}\left(e_{p}\right)^{*} \psi_{1}(x)=\eta\left\{\psi_{1}\left(e_{p}^{*} \tau_{n}(x)\right)\right\}=\eta\left\{\psi_{2}\left(e_{p}^{*} \tau_{n}(x)\right)\right\}=\psi_{2}\left(e_{p}\right)^{*} \psi_{2}(x) .
$$

Hence

$$
\psi_{2}(x)=c_{p} \psi_{1}(x), x \in \varepsilon_{p}(\mathfrak{A}), p \in H_{1}\left(=H_{2}\right) .
$$

For $p_{1}, p_{2} \in H_{1}$, we have

$$
\begin{aligned}
& e_{p_{1}}^{*} \tau_{n}\left(e_{p_{2}}\right) \in \varepsilon_{q}(\mathfrak{Q})\left(q=p_{1}^{-1} p_{2}\right), \\
& \eta\left\{\psi_{i}\left(e_{p_{1}} \tau_{n}\left(e_{p_{2}}\right)\right)\right\}=\psi_{i}\left(e_{p_{1}}\right) \psi_{i}\left(e_{p_{2}}\right) \neq 0 .
\end{aligned}
$$


Hence

$$
q=p_{1}^{-1} p_{2} \in H,\left(c_{p_{1}}\right)^{-1} c_{p_{2}}=c_{q} \text {. }
$$

This shows that $H$ is a subgroup of $G^{\wedge}$ and $c_{p} \in H^{\wedge}$. Hence there exists $g \in G$ satisfying $c_{p}=p(g)$ for $p \in H$.

We now have

$$
\psi_{2}(x)=p(g) \psi_{1}(x)=\psi_{1}(g(x))
$$

firstly for $x \in \varepsilon_{p}(\mathfrak{U}), p \in H$, by (9.6), secondly for $x \in \varepsilon_{q}(\mathfrak{A}), q \notin H$, because $\psi_{1}(x)=$ $\psi_{2}(x)=0$, and hence for all $x \in \mathfrak{A}$.

If $\varphi^{\wedge}$ of Theorem 1(4) turns out to be either $\varrho_{t}$ (or $\varrho_{n t_{0}}$ ) ergodic or $\tau_{n}$ invariant for some sequence of *-automorphisms commuting with $G$ and satisfying (9.3) (such as *-automorphisms of spatial translations), then this proposition is applicable and $\varphi^{\wedge} \circ g, g \in G$, are only clustering extensions of $\varphi$ to $\mathfrak{A}$.

Let $G_{1}$ be the closure of $\left\{\alpha_{t} ; t \in \mathbb{R}\right\}$ in $G$ and $\varphi^{\sim}=\int_{G_{1}} \varphi^{\wedge} \circ g d_{1} g$ where $d_{1} g$ is the normalized Haar measure on $G_{1}$. If the time translation $\varrho_{t}$ has an asymptotic abelianness, then this $\varphi^{\sim}$ is ergodic and the above result is applicable, namely $\varphi^{\sim} \circ g, g \in G$, are unique extensions of $\varphi$ to clustering states of $\mathfrak{A}$.

\section{References}

1. Araki, H.: Publ. RIMS Kyoto Univ. 9, 1-44 (1973)

2. Haag, R., Kastler, D., Trych-Pohlmeyer,E. B.: Commun. math. Phys. 38, 173-193 (1974)

3. Takesaki, M.: Acta Math. 131, 79-121 (1973)

4. Araki,H., Kastler,D., Takesaki, M., Haag, R.: Extension of KMS states and chemical potential. Commun. math. Phys., in press (1977)

Communicated by G. Gallavotti

Received July 30, 1976 